\title{
Optimum Performance Boundaries of OSTBC Based AF-MIMO Relay System With Energy Harvesting Receiver
}

\author{
Batu Krishna Chalise, Senior Member, IEEE, Wing-Kin Ma, Senior Member, IEEE, \\ Yimin D. Zhang, Senior Member, IEEE, Himal A. Suraweera, Member, IEEE, and Moeness G. Amin, Fellow, IEEE
}

\begin{abstract}
This paper studies the optimum performance boundaries of a two-hop multi-antenna amplify-and-forward (AF) relay system with a multi-antenna energy harvesting $(\mathrm{EH})$ receiver. The source and relay nodes employ orthogonal space-time block codes for data transmission. When instantaneous channel state information (CSI) is available, we design joint optimal source and relay precoders to achieve different tradeoffs between the energy and information transfers, which are characterized by the boundary of the rate-energy (R-E) region. For this purpose, the optimization problem is formulated as a relaxed convex problem but its optimality is confirmed with a proof that rank-one optimal precoders can always be obtained. As a consequence, it is shown that the full-rate OSTBC, like the Alamouti code, can be employed for an arbitrary number of antennas at the transmit nodes (source and relay) and support up to seven simultaneously existing EH receivers. When only second order statistics of the CSI is available, the tradeoff between outage probability (OP) and energy is characterized by the boundary of the $O P$-energy (OP-E) region. In this case, the precoder design problem is formulated using a convex upper bound approximation to the OP, since the exact OP expression is difficult for tractable optimization. Numerical results show that the OP-E region obtained with the upper bound of the OP is better than that with the approach based on maximization of the long-term average signal-to-noise ratio (SNR). The role of the different parameters such as average $S N R$, numbers of antennas, and spatial correlation on the boundaries of the $R-E$ and OP-E regions is demonstrated via simulations.
\end{abstract}

Index Terms-Energy harvesting, information and energy transfer, MIMO relay, orthogonal space-time block code.

Manuscript received November 05, 2012; revised March 24, 2013; accepted May 13,2013. Date of publication May 31, 2013; date of current version August 01,2013 . The associate editor coordinating the review of this manuscript and approving it for publication was Prof. Samson Lasaulce. The work of W.-K. Ma was supported in part by a Direct Grant of The Chinese University of Hong Kong (Project Code 2050506). Part of this work was presented at the IEEE ICASSP'12, Kyoto, Japan, March 2012

B. K. Chalise, Y. D. Zhang, and M. G. Amin are with the Center for Advanced Communications, Villanova University, Villanova, PA 19085 USA (e-mail: batu.chalise@villanova.edu; yimin.zhang@villanova.edu; moeness.amin@villanova.edu).

W.-K. Ma is with the Department of Electronic Engineering, Chinese University of Hongkong, Shatin, N.T., Hong Kong (e-mail: wkma@ieee.org).

H. A. Suraweera was with the Singapore University of Technology and Design, Singapore. He is now with the Department of Electrical and Electronic Engineering, University of Peradeniya, Peradeniya 20400, Sri Lanka (e-mail: himal@ee.pdn.ac.lk).

Color versions of one or more of the figures in this paper are available online at http://ieeexplore.ieee.

Digital Object Identifier 10.1109/TSP.2013.2265224

\section{INTRODUCTION}

$\mathbf{E}$ NERGY HARVESTING (EH) is the process by which energy from external sources (e.g., radio-frequency (RF) energy, solar power, thermal energy, wind energy) is captured and stored for energy-constrained devices. In particular, EH from radio waves, known as RF energy harvesting (RF-EH), is a promising technique that can be used for prolonging network operation time in energy-constrained networks, such as sensor networks, which are typically powered by small batteries and have limited life time [1]-[10]. The sources for RF-EH can be grouped into three general categories: intentional, anticipated ambient, and unknown ambient [11]. In [1]-[4], transmission strategies for EH nodes are studied, where the nodes harvest energy at some time instants and use the harvested energy for message transmission at some other time instants. The authors of [1] solve the problem of transmission time minimization, when the time instants and the amounts of data and energy, respectively, to transmit and harvest, are known. The short-term throughput is maximized in [2] for an EH system under the constraints on energy replenishment process and energy storage capacity of batteries. The relation between this optimization problem and transmission time minimization considered in [1] is demonstrated in [2], which also results into the solution of the latter problem. This design problem is extended in [3] to a realistic scenario of battery degradation over time by considering a time-varying battery capacity, and in [4], [5] to a two-hop relay channel, where both single-antenna source and relay nodes harvest energy. Gurakan et al. in [6] consider a scenario where the source node assists the relay by transferring some of its energy. The corresponding end-to-end throughput maximization problem with wireless energy transfer subject to energy causality at both source and relay nodes and data causality at the relay node is formulated as a convex optimization problem. Recently, a new protocol is proposed in [7], which computes the fraction of each time frame reserved for harvesting energy and the rest of the frame for concurrently transmitting message.

In [8]-[10], EH systems are studied from a different perspective, i.e., communication systems are designed to efficiently transmit both wireless power and information so that low-powered nodes that exist in the vicinity of these systems can harvest energy. In this context, the authors in [8] consider a threenode wireless multiple-input multiple-output (MIMO) broadcasting system in which the two receiver nodes harvest energy and decode information separately from the signals broadcast by the common transmitter. The transmitter tries to simultaneously maximize the information transfer to the intended receiver and energy transfer to the EH receiver. Notice that [8] extends 
the study of simultaneous information and energy transfer of [9], [10] from the single-input single-output (SISO) link in the co-located receiver (i.e., information and $\mathrm{EH}$ receivers are the same) case to the multi-antenna setup with both co-located and separated receivers. To the best of our knowledge, simultaneous transfer of energy and information for the MIMO relay system has not been addressed before, though optimal MIMO relay precoder designs have been solved for different scenarios (see [12] and the references therein) in the absence of the EH receiver.

In this paper, different from the previous contributions [1]-[10], we examine a wireless communication system, in which, two multi-antenna user terminals, an EH receiver and an information decoding (ID) receiver, with different requirements, namely, information and energy, are served by a common network of transmitters consisting of multi-antenna source and relay nodes. Therefore, the EH part of the considered system may be viewed as an intentional $\mathrm{EH}$, where the source and relay nodes are assisting the EH receiver to fulfill its energy requirement. The requirements for both energy and information arise, for example, when the ID-receiver with sufficient available power is ready for only receiving information, whereas the $\mathrm{EH}$ receiver, being an energy-constrained battery-powered device, is interested in harvesting energy so that it can charge its battery. The latter task is important since the EH receiver cannot enter into a communication phase without having enough power level. The source and relay nodes employ orthogonal space-time block codes (OSTBCs) [13] and precoders for data transmission. The relay operates in a half-duplex mode using an amplify-and-forward (AF) protocol [14]. Assuming perfect channel estimation at the receiving nodes, the relay/destination node of the two-hop system uses the maximum-ratio combining (MRC) technique for detecting/decoding the source signal. The EH receiver harvests energy from radio signals transmitted by both the source and relay nodes. Information transfer to the destination node and energy transfer to the $\mathrm{EH}$ receiver are optimally controlled by properly designing the source and relay precoders. Since our aim is to study the tradeoff between these transfers under different levels of transmit channel state information (CSI), the precoded OSTBC [15] is adopted, which can be considered to be a generalization of transmit beamforming $[16]^{1}$. Furthermore, optimal decoding in OSTBC-based MIMO systems can be significantly simplified. Specifically, by using MRC, the OSTBC-based MIMO transmission channel can be equivalently turned to parallel SISO channels. Notice that these benefits may be achieved without incurring rate-loss, for example, by using the Alamouti code [17] which is a full-rate OSTBC.

We investigate two scenarios, one assuming perfect CSI, whereas in the other, only the second-order statistics of CSI at the transmitting nodes (source and relay) are assumed to be available. For the former case, using the total power constraint ${ }^{2}$

\footnotetext{
${ }^{1}$ Although precoded OSTBC turns to transmit beamforming for rank-one precoders, the optimal design of source and relay beamformers proposed in [16] cannot be directly applied or easily extended to the system with an EH receiver.

${ }^{2}$ The precoder optimization with per-node individual power constraints (IPC) follows straightforwardly from the optimization with the sum power constraint (SPC). Accordingly, given the space constraint, we only consider the latter case. For a given total power budget, SPC allows more flexible power allocation between the transmit nodes depending on the instantaneous channel conditions. Consequently, SPC provides better performance than the per-node IPC [18]. It is assumed that the hardware of each node allows to use the total power.
}

of the source and relay, we design the source and relay precoders that maximize the rate for the intended receiver while keeping the energy transfer to the $\mathrm{EH}$ receiver above a certain predefined value. This predefined value is varied to obtain the boundary of the rate-energy (R-E) region which describes the tradeoffs between information rate and energy transfers [19]. In this context, the purpose of joint source and relay precoder optimization is to use the total power resource in an optimal way while serving the ID and EH receivers with two different objectives, namely, information transmission and energy harvesting, respectively. These different objectives render the underlying problem significantly different from conventional relay communication systems where the users compete for system resources with the same requirement, i.e., information transmission. One example to demonstrate such difference is that transmission from the relay alone may meet the objective of energy harvesting but not information transmission. To trade off between the two objectives under total power resource constraint, we use the R-E boundary and outage probability-energy (OP-E) boundary (this will be discussed later), which are defined as joint metrics of the information transmission and energy harvesting. It is clear that unless the joint optimization is carried out, the performance of the information transmission or energy harvesting, or both, will not achieve their optimal conditions. With these motivations behind joint precoder optimization, we reformulate the original optimization problem as a relaxed convex problem and confirm its optimality by proving that rank-one optimal source and relay precoders can be guaranteed. As a consequence, it is shown that the Alamouti code can be employed in a system with an arbitrary number of antennas at the transmit nodes. Furthermore, it is shown that the EH relay system with up to seven EH receivers can be supported by using the Alamouti code, without losing optimality of the relaxation.

In the second scenario where the instantaneous CSI is not known at the transmit nodes, the optimal precoders depend on the second-order statistics, such as covariance matrices of the channels. In this case, we characterize the performance of the underlying communication system using the tradeoff between the outage probability (OP) achieved at the destination and energy transferred to the EH receiver. This tradeoff is described by the boundary of the OP-E region. Because the exact expression of the OP is a complicated function of precoders, the design of joint optimal source and relay precoders becomes difficult. Therefore, we derive an upper bound approximation to the OP, which is used for formulating the precoder optimization problem. Due to the requirement of making the upper bound tight, this formulation, in addition to the precoders, involves optimization with respect to two more scalar variables. As a result, the corresponding optimization problem is solved by iteratively solving two convex sub-problems, where the sub-problem related with the optimization of scalar variables can be solved semi-analytically. Moreover, as in the perfect CSI case, the formulated problem is a relaxed optimization problem w.r.t. the precoders. However, unlike in the perfect CSI case, rank-one precoder solutions of the relaxed problem cannot not be guaranteed. Nonetheless, this does not lead to a loss in optimality (in the sense of minimizing the upper bound of $O P$ ) for a system that employs Alamouti codes and two transmit antennas at each node. The OP-E region obtained with this upper bound is com- 


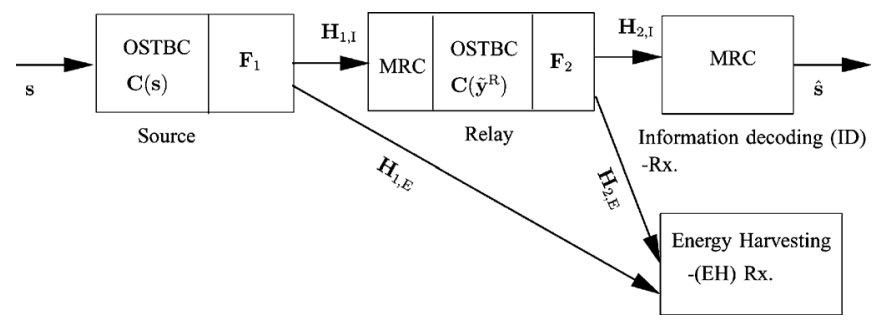

Fig. 1. Two-hop OSTBC based relay system with EH Rx.

pared with the approach which maximizes the long-term average signal-to-noise ratio (LTA-SNR).

The remaining of this paper is organized as follows. The system model is presented in Section II. In Sections III and IV, the precoder optimization methods are proposed for characterizing the R-E and OP-E regions, respectively. Simulations results are presented in Section V, whereas conclusions are made in Section VI.

Notations: Upper (lower) bold face letters will be used for matrices (vectors); $(\cdot)^{T},(\cdot)^{H}, \mathrm{E}\{\cdot\}, \mathbf{I}_{n}$ and $\|\cdot\|$ denote transpose, Hermitian transpose, mathematical expectation, $n \times n$ identity matrix, and Frobenius norm, respectively. $\operatorname{tr}(\cdot)$, $\operatorname{vec}(\cdot), \mathcal{C}^{M \times M}, \otimes$ and $\mathbf{A} \succeq 0$ denote the matrix trace operator, vectorization operator, space of $M \times M$ matrices with complex entries, the Kronecker product and positive semidefiniteness of $\mathbf{A}$, respectively. $[\mathbf{x}]_{k}$ stands for $k$ th element of the vector $\mathbf{x}$.

\section{System MODEL}

We consider a system shown in Fig. 1, which consists of a multi-antenna two-hop relaying system with an $n_{\mathrm{s}}$-sensor source, an $n_{\mathrm{r}}$-sensor relay, and an $n_{\mathrm{d}}$-sensor destination (also referred to as ID receiver), and an $n_{\mathrm{e}}$-sensor EH receiver. The purpose of the relay node is to support reliable information transfer from the source to the ID receiver. In particular, considering that the ID receiver lies in the shadowed area seen by the source node, we assume that there is no direct link between the source and the ID receiver. As such, the source has to rely on the relay node to extend the coverage. Consider that the source and relay are fixed infrastructure nodes in an LTE-advanced (LTE-A) system with the total available power as specified in [20]. This power is shared among these nodes, according to the optimization schemes to be presented in the following sections. The EH receiver is assumed to be not too far away from the source and relay nodes and have LoS with them. Depending on the power sharing between these nodes, the EH receiver can receive an order of several $\mu \mathrm{W}$ of RF power from the source and (or) relay, even if the source-EH receiver (S-EH) and relay-EH receiver $(\mathrm{R}-\mathrm{EH})$ distances are about $100 \mathrm{~m}$. Measurement results also indicate that typical power levels at $25 \mathrm{~m}$ to 100 $\mathrm{m}$ distances from GSM base stations reach several $\mu \mathrm{W} / \mathrm{cm}^{2}$ [Chapter 3, [21]]. Energy harvesting from such levels of RF power [Chapter 3, [21]], [22] is useful in today's technology for a sensor node to operate environment sensing and transmit information. Our objective is to provide a joint source and relay precoder optimization for simultaneous energy and power transfer to the EH and ID receivers, respectively. The proposed optimization framework provides a general approach, meaning that it results in a special and simplified optimization problem when the EH receiver harvests energy using signals from only one of the nodes (e.g., when it is far from either the source or the relay node).

Since the relay operates in a half-duplex mode, signal transmissions over source-relay (S-R) and relay-ID receiver (R-ID) channels take place in two phases. In the first phase, the source encodes the input signal using the OSTBC, precodes the encoded signal and transmits the resulting signal to the relay. The $n_{\mathrm{r}} \times T$ matrix of received signal samples at the relay can be given by

$$
\mathbf{Y}_{\mathrm{r}}=\mathbf{H}_{1, \mathrm{I}} \mathbf{F}_{1} \mathbf{C}(\mathbf{s})+\mathbf{N}_{\mathrm{r}}
$$

where $\mathbf{s}=\left[s_{1}, \ldots, s_{K}\right]^{T}$ is $K \times 1$ information-bearing complex symbol vector, $\mathbf{C}(\mathbf{s})$ is the $B \times T$ OSTBC matrix formed from $\mathbf{s}, T$ is the number of time periods used for transmitting $\mathbf{s}, B$ is the spatial dimension of the OSTBC, $\mathbf{H}_{1, \mathrm{I}} \in \mathcal{C}^{n_{\mathrm{r}} \times n_{\mathrm{s}}}$ is the S-R MIMO channel, $\mathbf{F}_{1} \in \mathcal{C}^{n_{\mathrm{s}} \times B}$ is the source precoder, and $\mathbf{N}_{\mathrm{r}} \in \mathcal{C}^{n_{\mathrm{r}} \times T}$ is the matrix of zero-mean circularly symmetric complex Gaussian (ZMCSCG) elements with variance $\tilde{\sigma}_{1}^{2}$. It is assumed that $\left\{s_{k}\right\}_{k=1}^{K}$ are chosen from signal constellations with $\mathrm{E}\left\{\left|s_{k}\right|^{2}\right\}=1$. Due to the orthogonality of the OSTBC, $\mathbf{C}(\mathbf{s})$ fulfills the property $\mathbf{C}(\mathbf{s}) \mathbf{C}^{H}(\mathbf{s})=a\|\mathbf{s}\|^{2} \mathbf{I}_{B}$, where the constant $a$ depends on the chosen OSTBC matrix (e.g., $a=$ 1 for the Alamouti code [17]). The power transmitted by the source during the first phase of the transmission is given by

$$
\begin{aligned}
P_{\mathrm{s}} & =\mathrm{E}\left\{\operatorname{tr}\left(\mathbf{F}_{1} \mathbf{C}(\mathbf{s}) \mathbf{C}^{H}(\mathbf{s}) \mathbf{F}_{1}^{H}\right)\right\} \\
& =a \mathrm{E}\left\{\|\mathbf{s}\|^{2}\right\} \operatorname{tr}\left(\mathbf{F}_{1} \mathbf{F}_{1}^{H}\right)=a K \operatorname{tr}\left(\mathbf{F}_{1} \mathbf{F}_{1}^{H}\right)
\end{aligned}
$$

where $\mathbf{H}_{1, \mathrm{E}} \in \mathcal{C}^{n_{\mathrm{e}} \times n_{\mathrm{s}}}$ is the S-EH MIMO channel. Notice that the $\mathrm{EH}$ receiver can harvest energy from the received RF signal without requiring to convert it to the base band signal, for example, using the rectenna architecture [21], [23]. Towards this end, the total harvested energy (also energy to be stored) is approximated to be linearly proportional to the received baseband signal power with some conversion efficiency $0<\mu \leq 1$ [23]. Thus, the energy harvested by the EH receiver during the first phase of the two-hop transmission can be expressed as

$$
\begin{aligned}
P_{\mathrm{e}, 1} & =\mu \mathrm{E}\left\{\operatorname{tr}\left(\mathbf{H}_{1, \mathrm{E}} \mathbf{F}_{1} \mathbf{C}(\mathbf{s}) \mathbf{C}^{H}(\mathbf{s}) \mathbf{F}_{1}^{H} \mathbf{H}_{1, \mathrm{E}}^{H}\right)\right\} \\
& =\tilde{a} \operatorname{tr}\left(\mathbf{H}_{1, \mathrm{E}} \mathbf{F}_{1} \mathbf{F}_{1}^{H} \mathbf{H}_{1, \mathrm{E}}^{H}\right),
\end{aligned}
$$

where $\tilde{a}=\mu a K$. It is important to emphasize that the assumed linear relationship between the harvested energy and received baseband signal power is standard (see also references in [21], [23]) and sufficiently accurate since the harvested energy due to the antenna noise and rectifier noise is a small constant. Moreover, since $\mu$ is related to a circuit that converts the received RF signal into harvested energy, the precoder design problems to be solved in this paper are not affected, at least from the perspective of optimization methodology. Due to the application of the OSTBC at the source and the MRC scheme at the relay during the first phase of signal transmission, the S-R MIMO channel is decoupled into $K$ parallel SISO channels. Thus, the signal received by the relay on the $k$ th S-R SISO channel is given by [24]

$$
y_{k}^{\mathrm{R}}=\left\|\mathbf{H}_{1, \mathrm{I}} \mathbf{F}_{1}\right\| s_{k}+n_{1, k}, \quad k \in\{1, \ldots, K\}
$$


where $n_{1, k} \sim \mathcal{N}_{\mathcal{C}}\left(0, \sigma_{1}^{2}\right)$ is the additive Gaussian noise at the relay for the $k$ th S-R SISO channel and $\sigma_{1}^{2}=\tilde{\sigma}_{1}^{2} / a$. The relay normalizes $\left\{y_{k}^{\mathrm{R}}\right\}_{k=1}^{K}$ yielding

$$
\tilde{y}_{k}^{\mathrm{R}}=\frac{y_{k}^{\mathrm{R}}}{\sqrt{\mathrm{E}\left\{\left|y_{k}^{\mathrm{R}}\right|^{2}\right\}}}=\frac{\left\|\mathbf{H}_{1, \mathrm{I}} \mathbf{F}_{1}\right\| s_{k}+n_{1, k}}{\sqrt{\left\|\mathbf{H}_{1, \mathrm{I}} \mathbf{F}_{1}\right\|^{2}+\sigma_{1}^{2}}} .
$$

The relay then employs $\operatorname{OSTBC}^{3}$ to encode $\left\{\tilde{y}_{k}^{\mathrm{R}}\right\}_{k=1}^{K}$ and precodes the resulting OSTBC encoded signal. The output of the relay is thus given by $\mathbf{Y}_{\text {ro }}=\mathbf{F}_{2} \mathbf{C}(\tilde{\mathbf{y}})$ where $\mathbf{F}_{2} \in \mathcal{C}^{n_{\mathrm{r}} \times B}$ is the relay precoder, $\tilde{\mathbf{y}}=\left[\tilde{y}_{1}^{\mathrm{R}}, \ldots, \tilde{y}_{K}^{\mathrm{R}}\right]^{T}, \mathbf{C}(\tilde{\mathbf{y}}) \in \mathcal{C}^{B \times T}$ is the OSTBC obtained after encoding $\tilde{\mathbf{y}}$ and satisfies the relation $\mathbf{C}(\tilde{\mathbf{y}}) \mathbf{C}^{H}(\tilde{\mathbf{y}})=a\|\tilde{\mathbf{y}}\|^{2} \mathbf{I}_{B}$. The transmit power of the relay and the energy harvested by the $\mathrm{EH}$ receiver during the second phase can be thus, respectively, given by

$$
P_{\mathrm{r}}=a K \operatorname{tr}\left(\mathbf{F}_{2} \mathbf{F}_{2}^{H}\right), P_{\mathrm{e}, 2}=\tilde{a} \operatorname{tr}\left(\mathbf{H}_{2, \mathrm{E}} \mathbf{F}_{2} \mathbf{F}_{2}^{H} \mathbf{H}_{2, \mathrm{E}}^{H}\right)
$$

where $\mathbf{H}_{2, \mathrm{E}}$ is the R-EH MIMO channel. The $n_{\mathrm{d}} \times T$ matrix of signal samples received at the ID receiver during the second phase of transmission can be written as

$$
\mathbf{Y}_{\mathrm{d}}=\mathbf{H}_{2, \mathrm{I}} \mathbf{F}_{2} \mathbf{C}(\tilde{\mathbf{y}})+\mathbf{N}_{\mathrm{d}}
$$

where $\mathbf{H}_{2, \mathrm{I}} \in \mathcal{C}^{n_{\mathrm{d}} \times n_{\mathrm{r}}}$ is the R-ID MIMO channel and $\mathbf{N}_{\mathrm{d}} \in$ $\mathcal{C}^{n_{\mathrm{d}} \times T}$ is the matrix of ZMCSCG elements with variance $\tilde{\sigma}_{2}^{2}$. The ID receiver uses MRC to detect the source signals. Due to the application of the OSTBC at the relay and MRC at the ID receiver, the R-ID MIMO channel also turns into $K$ parallel SISO channels. Thus, the signal received by the ID receiver on the $k$ th R-ID SISO channel can be expressed as

$$
y_{k, \mathrm{~d}}=\left\|\mathbf{H}_{2, \mathrm{I}} \mathbf{F}_{2}\right\| \tilde{y}_{k}^{\mathrm{R}}+n_{2, k}
$$

where $n_{2, k} \sim \mathcal{N}_{\mathcal{C}}\left(0, \sigma_{2}^{2}\right)$ is the additive Gaussian noise at the ID receiver for the $k$ th channel and $\sigma_{2}^{2}=\tilde{\sigma}_{2}^{2} / a$. With the help of (5), (8) can be written as

$$
y_{k, \mathrm{~d}}=\frac{\left\|\mathbf{H}_{2, \mathrm{I}} \mathbf{F}_{2}\right\|\left\|\mathbf{H}_{1, \mathrm{I}} \mathbf{F}_{1}\right\| s_{k}+\left\|\mathbf{H}_{2, \mathrm{I}} \mathbf{F}_{2}\right\| n_{1, k}}{\sqrt{\left\|\mathbf{H}_{1, \mathrm{I}} \mathbf{F}_{1}\right\|^{2}+\sigma_{1}^{2}}}+n_{2, k} .
$$

The signal-to-noise ratio (SNR) at the ID receiver can be expressed as

$$
\begin{aligned}
\gamma & =\frac{\left\|\mathbf{H}_{2, \mathrm{I}} \mathbf{F}_{2}\right\|^{2}\left\|\mathbf{H}_{1, \mathrm{I}} \mathbf{F}_{1}\right\|^{2}}{\left\|\mathbf{H}_{2, \mathrm{I}} \mathbf{F}_{2}\right\|^{2} \sigma_{1}^{2}+\left\|\mathbf{H}_{1, \mathrm{I}} \mathbf{F}_{1}\right\|^{2} \sigma_{2}^{2}+\sigma_{1}^{2} \sigma_{2}^{2}} \\
& =\frac{\gamma_{1} \gamma_{2}}{\gamma_{1}+\gamma_{2}+1}
\end{aligned}
$$

where $\gamma_{i}=\frac{\left\|\mathbf{H}_{i, \mathbf{I}} \mathbf{F}_{i}\right\|^{2}}{\sigma^{2}}$ for $i=1,2$. Equation (10) shows that the end-to-end SNR is a monotonically increasing function of

\footnotetext{
${ }^{3}$ For simplicity and notational convenience, it is assumed that the source and relay nodes use the same OSTBC. Since our aim is to use OSTBC without incurring rate loss, the available OSTBC for both source and relay nodes is, in fact, the Alamouti code. Because the employed OSTBCs are precoded, the aforementioned assumption does not result in any restriction on $n_{\mathrm{r}}$ and $n_{\mathrm{s}}$, except that $\left\{n_{\mathrm{r}}, n_{\mathrm{s}}\right\} \geq 2$.
}

the SNRs of S-R and R-ID links. The structure of this expression is the key for making the optimal precoder design problem tractable through an efficient approach.

\section{Full CSI CASE: R-E REgION}

In this section, we design optimal source and relay precoders considering that all channels are slowly time-varying, and thus, the instantaneous CSI of all channels can be obtained with negligible uncertainty. In order to obtain the transmit CSI of the S-R and R-ID channels, CSI acquisition methods based either on feedback (for frequency-division duplex transmission) or reciprocity (for time-division duplex systems) approach can be employed [25]. The EH receiver may use a small fraction of its $\mathrm{EH}$ time period to transmit control signals, which the source and relay nodes use to estimate the transmit CSI of the S-EH and R-EH channels, respectively. Although this requirement is motivated from the goal of achieving the best performance, it can be simplified or relaxed by using the precoder design based on only the second-order statistics of the channels (cf. Section IV). For better exposition of the concept behind R-E tradeoff, we first determine joint optimal $\mathbf{F}_{1}$ and $\mathbf{F}_{2}$ that maximize separately the average energy transfer to the $\mathrm{EH}$ receiver and the information transfer to the ID receiver. We then consider the problem of optimizing precoders when both receivers are present.

\section{A. Optimization With Only EH Receiver}

Consider the MIMO links from the source and relay to the $\mathrm{EH}$ receiver, when the ID receiver is not present. In this case, the objective is to design $\mathbf{F}_{1}$ and $\mathbf{F}_{2}$ to maximize the total power $P_{\mathrm{e}, 1}+P_{\mathrm{e}, 2}$ received at the $\mathrm{EH}$ receiver. This design problem can be formulated as

$$
\begin{aligned}
& \mathcal{P}_{1}: \max _{\mathbf{F}_{1}, \mathbf{F}_{2}} \operatorname{tr}\left(\mathbf{H}_{1, \mathrm{E}} \mathbf{F}_{1} \mathbf{F}_{1}^{H} \mathbf{H}_{1, \mathrm{E}}^{H}\right)+\operatorname{tr}\left(\mathbf{H}_{2, \mathrm{E}} \mathbf{F}_{2} \mathbf{F}_{2}^{H} \mathbf{H}_{2, \mathrm{E}}^{H}\right) \text { s.t. } \\
& \operatorname{tr}\left(\mathbf{F}_{1} \mathbf{F}_{1}^{H}\right)+\operatorname{tr}\left(\mathbf{F}_{2} \mathbf{F}_{2}^{H}\right) \leq P_{\mathrm{T}}
\end{aligned}
$$

where the constant $\tilde{a}$ is omitted from the objective function, and $P_{\mathrm{T}}$ is given by $P_{\mathrm{T}}=\frac{\tilde{P}_{\mathrm{T}}}{a K}$, where $\tilde{P}_{\mathrm{T}}$ is the total power available for the source and relay. Let the eigen-decomposition (ED) of $\mathbf{H}_{i, \mathrm{E}}^{H} \mathbf{H}_{i, \mathrm{E}}$ be given by $\mathbf{H}_{i, \mathrm{E}}^{H} \mathbf{H}_{i, \mathrm{E}}=\mathbf{U}_{\mathbf{H}_{i, \mathrm{E}}} \boldsymbol{\Lambda}_{\mathbf{H}_{i, \mathrm{E}}} \mathbf{U}_{\mathbf{H}_{i, \mathrm{E}}}^{H}$ with eigenvalues $\lambda_{k}^{\mathbf{H}_{i, \mathrm{E}}}\left(k=1, \ldots, r_{i, \mathrm{E}} \triangleq \operatorname{rank}\left(\mathbf{H}_{i, \mathrm{E}}\right)\right)$, in the non-increasing order, where $i=1,2$. Let $\left\{\mathbf{u}_{i, \mathrm{E}}\right\}_{i=1}^{2}$ be the column vectors of $\left\{\mathbf{U}_{\mathbf{H}_{i, \mathrm{E}}}\right\}_{i=1}^{2}$ corresponding to $\left\{\lambda_{1}^{\overline{\mathbf{H}}_{i, \mathrm{E}}}\right\}_{i=1}^{2}$. Then, we have the following proposition.

Proposition 1: The optimal solutions to $\mathcal{P}_{1}$ are given by

$$
\begin{aligned}
& \tilde{\mathbf{F}}_{i}= {\left[\sqrt{P_{\mathrm{T}}} \mathbf{u}_{i, \mathrm{E}}, \mathbf{0}, \ldots, \mathbf{0}\right], \tilde{\mathbf{F}}_{j}=[\mathbf{0}, \ldots, \mathbf{0}] } \\
& \text { if } \lambda_{1}^{\mathbf{H}_{i, \mathrm{E}}} \geq \lambda_{1}^{\mathbf{H}_{j, \mathrm{E}}}, \text { for } i \neq j, i, j \in\{1,2\} .
\end{aligned}
$$

Proof: For brevity, the proof for this proposition is summarized as follows. Substituting $\mathbf{H}_{i, \mathrm{E}}^{H} \mathbf{H}_{i, \mathrm{E}}=\mathbf{U}_{\mathbf{H}_{i, \mathrm{E}}} \boldsymbol{\Lambda}_{\mathbf{H}_{i, \mathrm{E}}} \mathbf{U}_{\mathbf{H}_{i, \mathrm{E}}}^{H}$, the ED of $\mathbf{F}_{i} \mathbf{F}_{i}^{H}$ into $\mathcal{P}_{1}$ and applying [(9.H.1.g.7), [26]], the matrix-variate optimization problem $\mathcal{P}_{1}$ can be converted to a scalar-valued linear programming (LP) problem [27]. The solution of this LP results in the solution (12).

\section{B. Optimization With Only ID Receiver}

Consider the two-hop MIMO relay link from the source to the ID receiver without the presence of the EH receiver. The 
optimal $\mathbf{F}_{1}$ and $\mathbf{F}_{2}$ that maximize the information rate over the two-hop MIMO channel can be obtained by solving the following problem

$$
\begin{aligned}
& \mathcal{P}_{2}: R_{\max } \triangleq \max _{\mathbf{F}_{1}, \mathbf{F}_{2}, \gamma_{1}, \gamma_{2}} \frac{R_{\mathrm{c}}}{2} \ln _{2}\left(1+\frac{\gamma_{1} \gamma_{2}}{\gamma_{1}+\gamma_{2}+1}\right) \text { s.t. } \\
& \gamma_{1}=\frac{\left\|\mathbf{H}_{1, \mathrm{I}} \mathbf{F}_{1}\right\|^{2}}{\sigma_{1}^{2}}, \gamma_{2}=\frac{\left\|\mathbf{H}_{2, \mathrm{I}} \mathbf{F}_{2}\right\|^{2}}{\sigma_{2}^{2}} \\
& \operatorname{tr}\left(\mathbf{F}_{1} \mathbf{F}_{1}^{H}\right)+\operatorname{tr}\left(\mathbf{F}_{2} \mathbf{F}_{2}^{H}\right) \leq P_{\mathrm{T}}
\end{aligned}
$$

where $R_{\mathrm{c}}$ is the code rate of the OSTBC (i.e., $R_{\mathrm{c}}=\frac{K}{T}$ ) and the factor $\frac{1}{2}$ is due to the half-duplex relay. Let the $\mathrm{ED}$ of $\mathbf{H}_{j, \mathrm{I}}^{H} \mathbf{H}_{j, \mathrm{I}}$ be given by $\mathbf{H}_{j, \mathrm{I}}^{H} \mathbf{H}_{j, \mathrm{I}}=\mathbf{U}_{\mathbf{H}_{j, 1}} \boldsymbol{\Lambda}_{\mathbf{H}_{j, 1}} \mathbf{U}_{\mathbf{H}_{j, \mathrm{I}}}^{H}$ with the eigenvalues $\lambda_{k}^{\mathbf{H}_{j, \mathrm{I}}}\left(k=1, \ldots, r_{j, \mathrm{I}} \triangleq \operatorname{rank}\left(\mathbf{H}_{j, \mathrm{I}}\right)\right)$ in the non-increasing order, where $j=1,2$. Let $\left\{\mathbf{u}_{j, \mathrm{I}}\right\}_{j=1}^{2}$ be the column vectors of $\left\{\mathbf{U}_{\mathbf{H}_{j, 1}}\right\}_{j=1}^{2}$ corresponding to $\left\{\lambda_{1}^{\mathbf{H}_{j, 1}}\right\}_{j=1}^{2}$. Following Proposition 1, we can prove that the optimal choices for $\mathbf{F}_{i}$ in $\mathcal{P}_{2}$ are

$$
\overline{\mathbf{F}}_{i}=\left[\sqrt{a_{i}} \mathbf{u}_{i, \mathrm{I}}, \mathbf{0}, \ldots, \mathbf{0}\right], i=1,2
$$

where $\left\{a_{i} \geq 0\right\}$ need to be optimized for $\mathcal{P}_{2}$. This leads to the following scalar-valued optimization problem for $\mathcal{P}_{2}$.

$$
\begin{aligned}
\overline{\mathcal{P}}_{2}: R_{\max } & \triangleq \max _{a_{1}, a_{2}} \frac{R_{\mathrm{c}}}{2} \ln _{2}\left(1+\frac{a_{1} a_{2} \tilde{\lambda}_{1}^{\mathbf{H}_{1, \mathrm{I}}} \tilde{\lambda}_{1}^{\mathbf{H}_{2, \mathrm{I}}}}{a_{1} \tilde{\lambda}_{1}^{\mathbf{H}_{1, \mathrm{I}}}+a_{2} \tilde{\lambda}_{1}^{\mathbf{H}_{2, \mathrm{I}}}+1}\right) \text { s.t. } \\
a_{1}+a_{2} & \leq P_{\mathrm{T}}
\end{aligned}
$$

where $\tilde{\lambda}_{1}^{\mathbf{H}_{1, \mathrm{I}}}=\frac{\lambda_{1}^{\mathbf{H}_{1, \mathrm{I}}}}{\sigma_{1}^{2}}$ and $\tilde{\lambda}_{1}^{\mathbf{H}_{2, \mathrm{I}}}=\frac{\lambda_{1}^{\mathbf{H}_{2, \mathrm{I}}}}{\sigma_{2}^{2}}$. It is easy to verify that the inequality $a_{1}+a_{2} \leq P_{\mathrm{T}}$ satisfies with equality at the optimality of $\overline{\mathcal{P}}_{2}$. Thus, substituting $a_{1}=P_{\mathrm{T}}-a_{2}$ into (15) and solving the first-order partial derivative of the objective function of $\overline{\mathcal{P}}_{2}$ w.r.t. $a_{2}$, we obtain the following solution

$$
a_{2}=\frac{u_{1} \pm \sqrt{u_{1}\left(1+\tilde{\lambda}_{1}^{\mathbf{H}_{2,1}} P_{\mathrm{T}}\right)}}{\tilde{\lambda}_{1}^{\mathbf{H}_{1, \mathrm{I}}}-\tilde{\lambda}_{1}^{\mathbf{H}_{2, \mathrm{I}}}}
$$

where $u_{1}=1+\tilde{\lambda}_{1}^{\mathbf{H}_{1,1}} P_{\mathrm{T}}$. The value of $a_{2}$ with "_" is taken to guarantee that $0 \leq a_{2} \leq P_{\mathrm{T}}$. Then, $a_{1}$ is obtained from $a_{1}=P_{\mathrm{T}}-a_{2}$. When $\tilde{\lambda}_{1}^{\mathbf{H}_{1,1}}=\tilde{\lambda}_{1}^{\mathbf{H}_{2,1}}$, it can be readily shown that $a_{1}=a_{2}=\frac{P_{\mathrm{T}}}{2}$. Thus, the optimal solutions for $\mathcal{P}_{2}$ are derived.

\section{Optimization With Both ID and EH Receivers}

We now consider the case where both the EH and ID receivers are present. In this case, our objective is to find the optimal transmission strategy for simultaneous wireless energy and information transfer. For this purpose, we use the rate-energy (R-E) region which characterizes all the achievable rate and energy pairs for a given total power constraint of source and relays. We define the R-E region as

$$
\begin{aligned}
& \mathcal{C}_{\mathrm{R}-\mathrm{P}}\left(P_{\mathrm{T}}\right) \triangleq\left\{(R, P): R \leq \frac{R_{\mathrm{c}}}{2} \ln _{2}\left(\frac{\left(\gamma_{1}+1\right)\left(\gamma_{2}+1\right)}{\gamma_{1}+\gamma_{2}+1}\right),\right. \\
& \gamma_{1}=\frac{\left\|\mathbf{H}_{1, \mathrm{I}} \mathbf{F}_{1}\right\|^{2}}{\sigma_{1}^{2}}, \gamma_{2}=\frac{\left\|\mathbf{H}_{2, \mathrm{I}} \mathbf{F}_{2}\right\|^{2}}{\sigma_{2}^{2}}, \\
& \left.P \leq\left\|\mathbf{H}_{1, \mathrm{E}} \mathbf{F}_{1}\right\|^{2}+\left\|\mathbf{H}_{2, \mathrm{E}} \mathbf{F}_{2}\right\|^{2},\left\|\mathbf{F}_{1}\right\|^{2}+\left\|\mathbf{F}_{2}\right\|^{2} \leq P_{\mathrm{T}}\right\} .
\end{aligned}
$$

Let $\left(R_{\mathrm{EH}}, P_{\max }\right)$ and $\left(R_{\max }, P_{\mathrm{ID}}\right)$ be the boundary points of this R-E region corresponding to the maximal energy and information transfers, respectively. The source and relay precoders for the former boundary point are given by (12), which yield maximum energy transfer of $P_{\max }=\tilde{a}\left(\left\|\mathbf{H}_{1, \mathrm{E}} \tilde{\mathbf{F}}_{1}\right\|^{2}+\left\|\mathbf{H}_{2, \mathrm{E}} \tilde{\mathbf{F}}_{2}\right\|^{2}\right)$ to the EH receiver and the information transfer of $R_{\mathrm{EH}}=0$ to the ID receiver. Note that no transfer of information to the ID receiver is obvious in this case, since the solution (12) means that either the source or the relay remains turned off. On the other hand, the source and relay precoders for the latter boundary point are given by (14) together with (16). With these precoders (i.e., $\overline{\mathbf{F}}_{1}$ and $\overline{\mathbf{F}}_{2}$ ), the information rate of $R_{\max }$ is achieved whereas the energy transferred to the $\mathrm{EH}$ receiver becomes $P_{\mathrm{ID}}=\tilde{a}\left(\left\|\mathbf{H}_{1, \mathrm{E}} \overline{\mathbf{F}}_{1}\right\|^{2}+\left\|\mathbf{H}_{2, \mathrm{E}} \overline{\mathbf{F}}_{2}\right\|^{2}\right)$. It can be easily seen that for $\bar{P} \leq P_{\mathrm{ID}}$, where $\bar{P} \geq 0$, the maximum rate $R_{\max }$ is achievable with the same $\overline{\mathbf{F}}_{1}$ and $\overline{\mathbf{F}}_{2}$ that achieve the R-E pair $\left(R_{\text {max }}, P_{\mathrm{ID}}\right)$. The remaining boundary of R-E region that needs to be characterized is over the interval $P_{\mathrm{ID}}<\bar{P}<P_{\max }$. For this purpose, we consider the following relaxed optimization problem with $\mathbf{W}_{1} \triangleq \mathbf{F}_{1} \mathbf{F}_{1}^{H} \succeq 0$ and $\mathbf{W}_{2} \triangleq \mathbf{F}_{2} \mathbf{F}_{2}^{H} \succeq 0$ :

$$
\begin{aligned}
& \mathcal{P}_{3}: \max _{\mathbf{W}_{1}, \mathbf{W}_{2}, \gamma_{1}, \gamma_{2}} \frac{R_{\mathrm{c}}}{2} \ln _{2}\left(1+\frac{\gamma_{1} \gamma_{2}}{\gamma_{1}+\gamma_{2}+1}\right) \text { s.t } \\
& \operatorname{tr}\left(\mathbf{H}_{1, \mathrm{I}}^{H} \mathbf{H}_{1, \mathrm{I}} \mathbf{W}_{1}\right) \geq \gamma_{1} \sigma_{1}^{2}, \operatorname{tr}\left(\mathbf{H}_{2, \mathrm{I}}^{H} \mathbf{H}_{2, \mathrm{I}} \mathbf{W}_{2}\right) \geq \gamma_{2} \sigma_{2}^{2}, \\
& \operatorname{tr}\left(\mathbf{H}_{1, \mathrm{E}}^{H} \mathbf{H}_{1, \mathrm{E}} \mathbf{W}_{1}\right)+\operatorname{tr}\left(\mathbf{H}_{2, \mathrm{E}}^{H} \mathbf{H}_{2, \mathrm{E}} \mathbf{W}_{2}\right) \geq \frac{\bar{P}}{\tilde{a}} \\
& \operatorname{tr}\left(\mathbf{W}_{1}+\mathbf{W}_{2}\right) \leq P_{\mathrm{T}}, \mathbf{W}_{1} \succeq 0, \mathbf{W}_{2} \succeq 0
\end{aligned}
$$

where the rank-constraints on $\mathbf{W}_{i}, \forall i$ are relaxed ${ }^{4}$. The constraints of the optimization problem (18) are convex [28], whereas the objective function is quasi-concave. The quasi-concavity of $f\left(\gamma_{1}, \gamma_{2}\right) \triangleq \ln _{2}\left(1+\frac{\gamma_{1} \gamma_{2}}{\gamma_{1}+\gamma_{2}+1}\right)$ can be proved by showing that the ordered determinants of the bordered Hessian matrix [29] of $f\left(\gamma_{1}, \gamma_{2}\right)$ alternate in signs. The derivations are skipped due to space constraints. However, the fact that the objective function of (18) increases monotonically with $\gamma$, which in turn is a monotonically increasing function of $\gamma_{i}, \forall i$, allows us to equivalently replace (18a) by $\min _{\left\{\mathbf{W}_{i}, \gamma_{i}, \forall i\right\}} \tilde{f}\left(\gamma_{1}, \gamma_{2}\right)$, where $\tilde{f}\left(\gamma_{1}, \gamma_{2}\right)$ is defined as $\tilde{f}\left(\gamma_{1}, \gamma_{2}\right) \triangleq\left[\frac{1}{\gamma_{1}}+\frac{1}{\gamma_{2}}+\frac{1}{\gamma_{1} \gamma_{2}}\right]$. As a result, without loss of any optimality, (18) can be expressed as

$$
\min _{\left\{\mathbf{W}_{i,}, \gamma_{i}, \forall i\right\}} \tilde{f}\left(\gamma_{1}, \gamma_{2}\right) \text { s.t. }(18 \mathrm{~b}),(18 \mathrm{c}),(18 \mathrm{~d}) \text {. }
$$

The objective function of (19) can be shown to be convex as follows. Since $\left\{\gamma_{i}\right\}$ are positive scalars, $\left\{\gamma_{i}^{-1}\right\}$ are convex. Moreover, since the Hessian matrix of the term $\frac{1}{\gamma_{1} \gamma_{2}}$ can be readily shown to be a positive semidefinite matrix, $\frac{1}{\gamma_{1} \gamma_{2}}$ is a convex function of $\gamma_{1}$ and $\gamma_{2}$. Since sum of convex functions is convex [28], (19) is a convex optimization problem. By solving (19) and recovering optimal $\left\{\mathbf{F}_{i}\right\}$ from optimal $\left\{\mathbf{W}_{i}\right\}$, it is principally possible to characterize the R-E region described by (17). However, since the ranks of $\left\{\mathbf{W}_{i}\right\}$ are relaxed in (18) (or (19)), this recovery may not be straightforward. Fortunately, we can verify that the optimal $\left\{\mathbf{F}_{i}\right\}$ can be recovered from the

\footnotetext{
${ }^{4}$ It is obvious that this relaxation is optimal when $\mathbf{F}_{1}$ and $\mathbf{F}_{2}$ are square matrices, i.e., when $B=n_{\mathrm{s}}=n_{\mathrm{r}}$. However, when Alamouti code is used for avoiding rate loss in a system with $\left\{n_{\mathrm{s}}, n_{\mathrm{r}}\right\}>2$, the optimality of the relaxation requires further investigation, which will be considered in Proposition 2.
} 
optimal $\left\{\mathbf{W}_{i}\right\}$ without any loss of optimality. The main idea is to utilize the Shapiro-Barvinok-Pataki (SBP) rank reduction result [30] derived in [31] for a semidefinite relaxation (SDR) of complex-valued non-convex quadratically constrained quadratic programs (QCQP). However, in order to apply SBP result to (18), we have to equivalently formulate (18) to a standard SDR formulation of QCQP. These contributions are reflected in the following proposition:

Proposition 2: Rank-one optimal solutions of $\left\{\mathbf{W}_{i}\right\}_{i=1}^{2}$ can always be obtained for the optimization problem (18).

Proof: Please refer to Appendix A.

We should note that the part of the proof of Proposition 2 is based on SBP result which is constructive, meaning that we not only know that the rank-one solution exists, it can also be algorithmically obtained when necessary [31]. This necessity depends on the solutions obtained from a semidefinite programming (SDP) solver in (18) and will be commented later. Note that, although (19) is convex, the term $\frac{1}{\gamma_{1} \gamma_{2}}$ makes it difficult to reformulate (19) to a suitable form so that a general convex optimization toolbox can be used for solving (19). Towards this end, the following proposition is formulated.

Proposition 3: The optimization problem (19) is equivalent to

$$
\begin{aligned}
& \mathcal{P}_{3}: \min _{\left\{\mathbf{W}_{i}, \gamma_{i}, u_{i}, \tau_{i}, \forall i\right\}, \tilde{t}} \tau_{1}+\tau_{2}+\tilde{t} \text { s.t } \\
& {\left[\begin{array}{cc}
\gamma_{1} & u_{1} \\
u_{1} & \tilde{t}
\end{array}\right] \succeq 0,\left[\begin{array}{cc}
1 & u_{2} \\
u_{2} & \gamma_{2}
\end{array}\right] \succeq 0,} \\
& u_{1}+u_{2} \geq\left\|\left[\sqrt{2}, u_{1}, u_{2}\right]^{T}\right\|, \\
& \tau_{i}+\gamma_{i} \geq\left\|\left[\sqrt{2}, \tau_{i}, \gamma_{i}\right]^{T}\right\|, \forall i \\
& (18 \mathrm{~b}),(18 \mathrm{c}),(18 \mathrm{~d}) .
\end{aligned}
$$

which is a convex optimization problem since (20a) is linear, (20b) consists of linear matrix inequalities, (20c) and (20d) are second-order cone constraints, and the constraints of (20e) are convex.

Proof: Please refer to Appendix B.

By solving the problem $\mathcal{P}_{3}$ (e.g., with $\mathrm{CVX}$ software [32]) for $P_{\mathrm{ID}}<\bar{P}<P_{\max }$, we obtain the optimal rate solutions that form the boundary of the R-E region over the interval $\left(R_{\mathrm{EH}}=0\right)<R<R_{\max }$. It is important to emphasize that, although (18) is equivalent to (20) and Proposition 2 shows that rank-one optimal solutions of $\left\{\mathbf{W}_{i}\right\}$ can always be obtained, an SDP solver (e.g., CVX) may not give always rank-one optimal $\left\{\mathbf{W}_{i}\right\}$. In such cases, a rank reduction algorithm, on the basis of which the SBP result is derived, can be applied to the solutions given by CVX. Interestingly, in our simulations (cf. Section V) of (20), we have not encountered a case in which CVX yields optimal solutions of $\left\{\mathbf{W}_{i}\right\}$ with ranks higher than one. We now complete this section with the following remarks:

- Remark 1: It is clear from the Proposition 2 that the optimal source and relay precoders are none other than the transmit beamformers. In other words, the performance obtained with the optimal solutions of $\left\{\mathbf{W}_{i}\right\}_{i=1}^{2}$ should be same as that obtained with the optimal source, relay and ID-receiver beamformers without OSTBCs. However, in contrast to the case of a MIMO relay system without an EH receiver [16], it is difficult to obtain these optimal beamformers without employing OSTBCs in our case.
- Remark 2: The use of general OSTBCs may incur rate loss. However, the result of Proposition 2 enables us to employ Alamouti codes, even if $\left\{n_{\mathrm{s}}, n_{\mathrm{r}}\right\}>2$. This is due to the fact that the main system equations (1) and (7) remain unaffected except that the ranks of $\mathbf{F}_{1}$ and $\mathbf{F}_{2}$ reduce to two.

- Remark 3: The Alamouti code can be used to support multiple EH receivers for any $n_{\mathrm{s}} \geq 2$. The maximum number of $E H$ receivers can be determined by extending the results of Appendix A. Assume that there are $Q \mathrm{EH}$ receivers. The corresponding rate-maximization problem with the energy transfer constraints is given by

$$
\begin{aligned}
& \max _{\left\{\mathbf{W}_{i}, \gamma_{i}, \forall i\right\}} \frac{R_{\mathrm{c}}}{2} \ln _{2}\left(1+\frac{\gamma_{1} \gamma_{2}}{\gamma_{1}+\gamma_{2}+1}\right) \text { s.t. } \\
& \operatorname{tr}\left(\mathbf{H}_{1, q, \mathrm{E}}^{H} \mathbf{H}_{1, q, \mathrm{E}} \mathbf{W}_{1}\right)+\operatorname{tr}\left(\mathbf{H}_{2, q, \mathrm{E}}^{H} \mathbf{H}_{2, q, \mathrm{E}} \mathbf{W}_{2}\right) \geq \frac{\bar{P}}{\tilde{a}}
\end{aligned}
$$

$q \in\{1, \ldots, Q\},(18 \mathrm{~b}),(18 \mathrm{~d})$

where $\mathbf{H}_{1, q, \mathrm{E}}$ and $\mathbf{H}_{2, q, \mathrm{E}}$ denote the MIMO channels between the source and the $q$ th $\mathrm{EH}$ receiver, and between the relay and the $q$ th $\mathrm{EH}$ receiver, respectively. Let $\left(\mathbf{W}_{1}^{*}, \mathbf{W}_{2}^{*}, \gamma_{1}^{*}, \gamma_{2}^{*}\right)$ be the optimal solution of $(21)$. Consider the following problem

$$
\begin{aligned}
& \min _{\left\{\mathbf{W}_{i}\right\}_{i=1}^{2}} \operatorname{tr}\left(\mathbf{W}_{1}\right)+\operatorname{tr}\left(\mathbf{W}_{2}\right) \text { s.t. } \\
& \operatorname{tr}\left(\mathbf{H}_{1, \mathrm{I}}^{H} \mathbf{H}_{1, \mathrm{I}} \mathbf{W}_{1}\right) \geq \gamma_{1}^{*} \sigma_{1}^{2}, \operatorname{tr}\left(\mathbf{H}_{2, \mathrm{I}}^{H} \mathbf{H}_{2, \mathrm{I}} \mathbf{W}_{2}\right) \geq \gamma_{2}^{*} \sigma_{2}^{2},
\end{aligned}
$$

(21b), (18d).

As an extension of the proof in Appendix A, it can be readily shown that for any optimal solution $\left(\hat{\mathbf{W}}_{1}, \hat{\mathbf{W}}_{2}\right)$ of (22), $\left(\hat{\mathbf{W}}_{1}, \hat{\mathbf{W}}_{2}, \gamma_{1}^{*}, \gamma_{2}^{*}\right)$ forms an optimal solution of $(21)$. By the SBP result based on rank reduction algorithm [31], (22) has an optimal solution $\left(\hat{\mathbf{W}}_{1}, \hat{\mathbf{W}}_{2}\right)$ such that

$$
\left(\operatorname{rank}\left(\hat{\mathbf{W}}_{1}\right)\right)^{2}+\left(\operatorname{rank}\left(\hat{\mathbf{W}}_{2}\right)\right)^{2} \leq 2+Q .
$$

Moreover, as a necessary condition, it remains true that $\operatorname{rank}\left(\hat{\mathbf{W}}_{1}\right) \geq 1$ and $\operatorname{rank}\left(\hat{\mathbf{W}}_{2}\right) \geq 1$ (cf. Appendix A). Because of this necessary condition and the inequality (23), it can be easily verified that as long as $Q \leq 7$, we have optimal solutions in (21) satisfying $\operatorname{rank}\left(\hat{\mathbf{W}}_{i}\right) \leq 2, \forall i$. For example, assume that $Q=8$ is possible. In this case, according to (23), we may obtain $\operatorname{rank}\left(\hat{\mathbf{W}}_{1}\right)=3$ and $\operatorname{rank}\left(\hat{\mathbf{W}}_{2}\right)=1 \operatorname{or} \operatorname{rank}\left(\hat{\mathbf{W}}_{1}\right)=1 \operatorname{and} \operatorname{rank}\left(\hat{\mathbf{W}}_{2}\right)=3$, i.e., we cannot guarantee that $\operatorname{rank}\left(\hat{\mathbf{W}}_{i}\right) \leq 2, \forall i$. Consequently, in such cases, the full-rate OSTBCs do not exist. Furthermore, the fact that $\operatorname{rank}\left(\hat{\mathbf{W}}_{i}\right) \leq 2, \forall i$ is guaranteed for $Q \leq 7$ means that the proposed OSTBC based precoder optimization can handle up to seven $\mathrm{EH}$ receivers without incurring rate reduction. The following corollary and remark are now in order.

Corollary: For $Q \leq 7$, optimal solutions with $\operatorname{rank}\left(\mathbf{W}_{i}\right) \leq 2, \forall i$ can always be obtained in (21).

Remark 4: From the results of Proposition 2, Remark 3 and the Corollary, it can be observed that smaller the number of EH receivers, higher is the probability that the optimal solutions of $\left\{\mathbf{W}_{i}\right\}$ are low-rank matrices. This observation can be explained intuitively as follows. 
The resource allocation problem (21) (or $\mathcal{P}_{3}$ for $Q=1$ ) is solved in terms of $\left\{\mathbf{W}_{i}\right\}$ by relaxing the constraints $\mathbf{W}_{i}=\mathbf{F}_{i} \mathbf{F}_{i}^{H}, \forall i$ or equivalently the rank constraints on $\left\{\mathbf{W}_{i}\right\}$. This means the feasible region of (21) is larger than that of the original problem in terms of $\left\{\mathbf{F}_{i}\right\}$. In other words, the possible maximum ranks of $\left\{\mathbf{W}_{i}\right\}$ can be viewed as degrees-of-freedom (DoF) available for (21). On the other hand, $R_{\max }$, which is an upper bound to $(21 \mathrm{a})$, is achieved when there is no $\mathrm{EH}$ receiver, and most notably with rank-one optimal solutions of $\left\{\mathbf{F}_{i}\right\}$ (equivalently rank-one $\left\{\mathbf{W}_{i}\right\}$ ). Due to these reasons, it may be argued that not all available DoF are required for attaining the maximum value of (21a). This means that, as the number of EH receivers increases, more DoF will be used up, i.e., the probability of having optimal solutions of $\left\{\mathbf{W}_{i}\right\}$ with higher ranks increases.

\section{Outage Probability-Energy (OP-E) Region}

In the previous section, the optimal precoders that characterize the boundary of the R-E region are designed assuming that the instantaneous CSI of channels are perfectly known. In particular, the assumption of having perfect instantaneous CSI at transmit nodes (source and relay) is idealistic due to the fact that these nodes in general have to rely on the CSI fed back by the relay and ID receiver, and the control signals transmitted by the $\mathrm{EH}$ node for computing the optimal precoders. In order to minimize the cost of CSI feedback and simplify (or relax) the requirement for the $\mathrm{EH}$ receiver to allocate some of its $\mathrm{EH}$ time period to transmit control signals, the precoders are designed using the knowledge of second-order statistics of the channels. Notice that, if the channel varies rapidly, this approach becomes somehow inevitable, since the optimal precoders designed on the basis of previously acquired CSI becomes outdated quickly (see [33] and the references therein). Towards this end, we aim to analyze the OP and examine the tradeoffs between the OP and energy transfer. We first derive the closed-form expression for the OP in terms of the precoders and the second-order statistics of channels. Since the precoder optimization based on the exact expression is nonconvex and unlikely to be tractable, we derive an upper bound approximation to the OP and propose a convex optimization framework for characterizing the boundary of the OP-E region.

All MIMO channels are considered to exhibit both transmit and receive side correlations. Using the Kronceker's model [34], these channels can be expressed as

$$
\mathbf{H}_{i, \mathrm{I}}=\mathbf{R}_{i, \mathrm{I}, \mathrm{r}}^{\frac{1}{2}} \mathbf{H}_{i, \mathrm{I}, \mathrm{w}} \mathbf{R}_{i, \mathrm{I}, \mathrm{t}}^{\frac{1}{2}}, \mathbf{H}_{i, \mathrm{E}}=\mathbf{R}_{i, \mathrm{E}, \mathrm{r}}^{\frac{1}{2}} \mathbf{H}_{i, \mathrm{E}, \mathrm{w}} \mathbf{R}_{i, \mathrm{E}, \mathrm{t}}^{\frac{1}{2}}, \forall i
$$

where $\mathbf{R}_{1, \mathrm{I}, \mathrm{r}} \in \mathcal{C}^{n_{\mathrm{r}} \times n_{\mathrm{r}}}$ and $\mathbf{R}_{1, \mathrm{I}, \mathrm{t}} \in \mathcal{C}^{n_{\mathrm{s}} \times n_{\mathrm{s}}}$ are, respectively, the receive and transmit correlation matrices for the S-R channel, whereas the respective receive and transmit side correlation matrices for the R-ID channels are $\mathbf{R}_{2, \mathrm{I}, \mathrm{r}} \in \mathcal{C}^{n_{\mathrm{d}} \times n_{\mathrm{d}}}$ and $\mathbf{R}_{2, \mathrm{I}, \mathrm{t}} \in \mathcal{C}^{n_{\mathrm{r}} \times n_{\mathrm{r}}}$. Similarly, $\mathbf{R}_{1, \mathrm{E}, \mathrm{r}} \in \mathcal{C}^{n_{\mathrm{e}} \times n_{\mathrm{e}}}$ and $\mathbf{R}_{1, \mathrm{E}, \mathrm{t}} \in \mathcal{C}^{n_{\mathrm{s}} \times n_{\mathrm{s}}}$ represent receive and transmit spatial correlation matrices for the S-EH channel, respectively, whereas the respective correlation matrices for the R-EH channel are represented by $\mathbf{R}_{2, \mathrm{E}, \mathrm{r}} \in \mathcal{C}^{n_{\mathrm{e}} \times n_{\mathrm{e}}}$ and
$\mathbf{R}_{2, \mathrm{E}, \mathrm{t}} \in \mathcal{C}^{n_{\mathrm{r}} \times n_{\mathrm{r}}}$. The entries of $\mathbf{H}_{i, \mathrm{I}, \mathrm{w}}$ and $\mathbf{H}_{i, \mathrm{E}, \mathrm{w}}, \forall i$, are assumed to be i.i.d. ZMCSCG random variables ${ }^{5}$. We define $\alpha_{1} \triangleq\left\|\mathbf{H}_{1, \mathrm{I}} \mathbf{F}_{1}\right\|^{2}$ and $\alpha_{2} \triangleq\left\|\mathbf{H}_{2, \mathrm{I}} \mathbf{F}_{2}\right\|^{2}$. Using the facts that $\operatorname{tr}\left(\mathbf{X}^{H} \mathbf{Y} \mathbf{X W}\right)=\operatorname{vec}(\mathbf{X})^{H}\left(\mathbf{W}^{T} \otimes \mathbf{Y}\right) \operatorname{vec}(\mathbf{X})$, $\operatorname{vec}(\mathbf{X Y Z})=\left(\mathbf{Z}^{T} \otimes \mathbf{X}\right) \operatorname{vec}(\mathbf{Y})[35]$ and after some simple steps, we get

$$
\begin{aligned}
& \alpha_{1}=\operatorname{vec}\left(\mathbf{H}_{1, \mathrm{I}, \mathrm{w}}\right)^{H} \underbrace{\mathbf{R}_{1, \mathrm{I}}^{\frac{1}{2}}\left[\mathbf{W}_{1}^{T} \otimes \mathbf{I}_{n_{\mathrm{r}}}\right] \mathbf{R}_{1, \mathrm{I}}^{\frac{1}{2}}}_{\mathbf{\Phi}_{1}} \operatorname{vec}\left(\mathbf{H}_{1, \mathrm{I}, \mathrm{w}}\right), \\
& \alpha_{2}=\operatorname{vec}\left(\mathbf{H}_{2, \mathrm{I}, \mathrm{w}}\right)^{H} \underbrace{\mathbf{R}_{2, \mathrm{I}}^{\frac{1}{2}}\left[\mathbf{W}_{2}^{T} \otimes \mathbf{I}_{n_{\mathrm{d}}}\right] \mathbf{R}_{2, \mathrm{I}}^{\frac{1}{2}}}_{\mathbf{\Phi}_{2}} \operatorname{vec}\left(\mathbf{H}_{2, \mathrm{I}, \mathrm{w}}\right)
\end{aligned}
$$

where $\mathbf{R}_{1, \mathrm{I}}=\mathbf{R}_{1, \mathrm{I}, \mathrm{t}}^{T} \otimes \mathbf{R}_{1, \mathrm{I}, \mathrm{r}}$ and $\mathbf{R}_{2, \mathrm{I}}=\mathbf{R}_{2, \mathrm{I}, \mathrm{t}}^{T} \otimes \mathbf{R}_{2, \mathrm{I}, \mathrm{r}}$.

\section{A. Exact OP Expression}

In this subsection, we derive a closed-form expression for the OP. Using the ED $\boldsymbol{\Phi}_{1}=\mathbf{U}_{1} \boldsymbol{\Lambda}_{1} \mathbf{U}_{1}^{H}$ and $\boldsymbol{\Phi}_{2}=\mathbf{U}_{2} \boldsymbol{\Lambda}_{2} \mathbf{U}_{2}^{H}$, we can write

$$
\begin{aligned}
& \alpha_{1}=\operatorname{vec}\left(\mathbf{H}_{1, \mathrm{I}, \mathrm{w}}\right)^{H} \mathbf{U}_{1} \boldsymbol{\Lambda}_{1} \mathbf{U}_{1}^{H} \operatorname{vec}\left(\mathbf{H}_{1, \mathrm{I}, \mathrm{w}}\right) \triangleq \tilde{\mathbf{h}}^{H} \boldsymbol{\Lambda}_{1} \tilde{\mathbf{h}}, \\
& \alpha_{2}=\operatorname{vec}\left(\mathbf{H}_{2, \mathrm{I}, \mathrm{w}}\right)^{H} \mathbf{U}_{2} \boldsymbol{\Lambda}_{2} \mathbf{U}_{2}^{H} \operatorname{vec}\left(\mathbf{H}_{2, \mathrm{I}, \mathrm{w}}\right) \triangleq \tilde{\mathbf{g}}^{H} \boldsymbol{\Lambda}_{2} \tilde{\mathbf{g}}
\end{aligned}
$$

where $\mathrm{U}_{1} \in \mathcal{C}^{n_{\mathrm{s}} n_{\mathrm{r}} \times n_{\mathrm{s}} n_{\mathrm{r}}}$ and $\mathrm{U}_{2} \in \mathcal{C}^{n_{\mathrm{d}} n_{\mathrm{r}} \times n_{\mathrm{d}} n_{\mathrm{r}}}$ are unitary matrices, and $\boldsymbol{\Lambda}_{1}=\operatorname{diag}\left(\lambda_{1}^{(1)}, \ldots, \lambda_{n_{\mathrm{s}} n_{\mathrm{r}}}^{(1)}\right)$ and $\boldsymbol{\Lambda}_{2}=\operatorname{diag}\left(\lambda_{1}^{(2)}, \ldots, \lambda_{n_{\mathrm{d}} n_{\mathrm{r}}}^{(2)}\right)$ are the diagonal matrices representing the eigenvalues of $\boldsymbol{\Phi}_{1}$ and $\boldsymbol{\Phi}_{2}$, respectively. Since $\mathbf{U}_{1}$ and $\mathbf{U}_{2}$ are unitary, and the elements of both $\operatorname{vec}\left(\mathbf{H}_{1, \mathrm{I}, \mathrm{w}}\right)$ and $\operatorname{vec}\left(\mathbf{H}_{2, \mathrm{I}, \mathrm{w}}\right)$ are i.i.d. ZMCSCG random variables, the entries of $\tilde{\mathbf{h}} \triangleq \mathbf{U}_{1}^{H} \operatorname{vec}\left(\mathbf{H}_{1, \mathrm{I}, \mathrm{w}}\right)$ and $\tilde{\mathbf{g}} \triangleq \mathbf{U}_{2}^{H} \operatorname{vec}\left(\mathbf{H}_{2, \mathrm{I}, \mathrm{w}}\right)$ remain i.i.d. ZMCSCG random. Let $L_{1} \leq n_{\mathrm{s}} n_{\mathrm{r}}$ and $L_{2} \leq n_{\mathrm{r}} n_{\mathrm{d}}$ be the number of non-zero $\left\{\lambda_{i}^{(1)}\right\}_{i=1}^{n_{\mathrm{s}} n_{\mathrm{r}}}$ and $\left\{\lambda_{k}^{(2)}\right\}_{k=1}^{n_{\mathrm{s}} n_{\mathrm{d}}}$, respectively. In this case, $\alpha_{1}$ and $\alpha_{2}$ can be further expressed as

$$
\alpha_{1}=\sum_{i=1}^{L_{1}} \lambda_{i}^{(1)}\left|\tilde{h}_{i}\right|^{2}, \alpha_{2}=\sum_{k=1}^{L_{2}} \lambda_{k}^{(2)}\left|\tilde{g}_{k}\right|^{2}
$$

where $\tilde{h}_{i}$ and $\tilde{g}_{i}$ are the $i$ th elements of $\tilde{\mathbf{h}}$ and $\tilde{\mathrm{g}}$. Since $\tilde{h}_{i}$ and $\tilde{g}_{i}$ are i.i.d. ZMCSCG with the unit variance, under the assumption that both the weights, $\left\{\lambda_{i}^{(1)}\right\}_{i=1}^{L_{1}}$ of $\alpha_{1}$ and $\left\{\lambda_{k}^{(2)}\right\}_{k=1}^{L_{2}}$ of $\alpha_{2}$, are different ${ }^{6},\left|\tilde{h}_{i}\right|^{2}$ and $\left|\tilde{g}_{i}\right|^{2}$ will be exponentially distributed with parameter 1 . This means that $\alpha_{1}$ and $\alpha_{2}$ are the weighted sum of the exponentially distributed random variables. Note that $\frac{\alpha_{1}}{\sigma_{1}^{2}}=\gamma_{1}$ and $\frac{\alpha_{2}}{\sigma_{2}^{2}}=\gamma_{2}$ represent the instantaneous SNRs of the S-R and R-ID links, respectively. The OP as a function of precoders is defined as

$$
P_{\mathrm{o}}\left(\mathbf{W}_{1}, \mathbf{W}_{2}\right)=\operatorname{Pr}\left\{\frac{1}{2} \ln _{2}\left(1+\frac{\gamma_{1} \gamma_{2}}{\gamma_{1}+\gamma_{2}+1}\right) \leq r_{0}\right\}
$$

where $r_{0}$ is the predefined threshold for rate in bits/channel use. For notational simplicity, we drop the dependence of $P_{\mathrm{o}}$ on $\mathbf{W}_{1}$ and $\mathbf{W}_{2}$, and use the notation $P_{\mathrm{o}}$ for $P_{\mathrm{o}}\left(\mathbf{W}_{1}, \mathbf{W}_{2}\right)$ in the rest of

\footnotetext{
${ }^{5}$ Without loss of generality, unit variance is assumed.

${ }^{6}$ In practice, this assumption is reasonable since small perturbations make these eigenvalues different with a negligible effect on the final OP.
} 
the paper. Let $\gamma_{0}$ be defined as $\gamma_{0} \triangleq 2^{2 r_{0}}-1$. Then, the closedform expression for $P_{\mathrm{o}}$ is obtained in the following proposition 7 .

Proposition 4: The OP, $P_{o}=\operatorname{Pr}\left\{\gamma \leq \gamma_{0}\right\}$, for the ID-receiver can be expressed as

$$
\begin{aligned}
P_{\mathrm{o}}=1-\sum_{i=1}^{L_{1}} \sum_{k=1}^{L_{2}} \tilde{a}_{i} \tilde{b}_{k} \mathrm{e}^{-\frac{\gamma_{0} \sigma_{1}^{2}}{\lambda_{i}^{(1)}}} \mathrm{e}^{-\frac{\gamma_{0} \sigma_{2}^{2}}{\lambda_{k}^{(2)}}} \frac{2 \tilde{\gamma}_{0} \sigma_{1} \sigma_{2}}{\sqrt{\lambda_{i}^{(1)} \lambda_{k}^{(2)}}} \\
\times K_{1}\left(\frac{2 \tilde{\gamma}_{0} \sigma_{1} \sigma_{2}}{\sqrt{\lambda_{i}^{(1)} \lambda_{k}^{(2)}}}\right)
\end{aligned}
$$

where $\tilde{a}_{i}=\lambda_{i}^{(1)} a_{i}, \tilde{b}_{k}=\lambda_{k}^{(2)} b_{k}$,

$$
a_{i}=\frac{\left(\lambda_{i}^{(1)}\right)^{L_{1}-2}}{\prod_{j=1, j \neq i}^{L_{1}} \lambda_{i}^{(1)}-\lambda_{j}^{(1)}}, b_{k}=\frac{\left(\lambda_{k}^{(2)}\right)^{L_{2}-2}}{\prod_{l=1, l \neq k}^{L_{2}} \lambda_{k}^{(2)}-\lambda_{l}^{(2)}},
$$

$\tilde{\gamma}_{0}=\sqrt{\gamma_{0}\left(\gamma_{0}+1\right)}$, and $K_{1}(\cdot)$ is the first-order modified Bessel's function of the second type.

Proof: Please refer to Appendix C.

It is worthwhile to mention that when $L_{1}=1$ and $L_{2}=1$, $P_{\mathrm{o}}$ reduces to the OP expression derived in [38].

\section{B. Upper Bound of the OP}

The derived OP expression (29) is a complicated function of precoders, and thus, any optimization problem based on this expression is difficult. Based on this observation, we derive the upper bound of the OP that results in a tractable precoder optimization problem. Towards this end, the main contribution of this subsection is included in the following proposition.

Proposition 5: The upper bound for $P_{\mathrm{o}}$ is given by

$$
\begin{aligned}
P_{\mathrm{o}} \leq & P_{\mathrm{o}, \mathrm{ub}} \triangleq \min _{\beta_{1}, \beta_{2}}\left\{\mathrm{e}^{\beta_{1} t_{\mathrm{c}}} \operatorname{det}\left(\mathbf{I}_{n_{\mathrm{r}} n_{\mathrm{s}}}+\frac{\beta_{1}}{\sigma_{1}^{2}} \boldsymbol{\Phi}_{1}\right)^{-1}\right. \\
& \left.+\mathrm{e}^{\beta_{2} t_{\mathrm{c}}} \operatorname{det}\left(\mathbf{I}_{n_{\mathrm{r}} n_{\mathrm{d}}}+\frac{\beta_{2}}{\sigma_{2}^{2}} \mathbf{\Phi}_{2}\right)^{-1}\right\} \\
= & \min _{\beta_{1}, \beta_{2}}\left\{\mathrm{e}^{\beta_{1} t_{\mathrm{c}}} \mathrm{e}^{-\ln \operatorname{det}\left(\mathbf{I}_{n_{\mathrm{r}} n_{\mathrm{s}}}+\frac{\beta_{1}}{\sigma_{1}^{2}} \boldsymbol{\Phi}_{1}\right)}\right. \\
& \left.+\mathrm{e}^{\beta_{2} t_{\mathrm{c}}} \mathrm{e}^{-\ln \operatorname{det}\left(\mathbf{I}_{n_{\mathrm{r}} n_{\mathrm{d}}}+\frac{\beta_{2}}{\sigma_{2}^{2}} \mathbf{\Phi}_{2}\right)}\right\}
\end{aligned}
$$

where $t_{\mathrm{c}}=\nu 2^{2 r_{0}}-1, \nu=2$ and $\left\{\beta_{i}>0\right\}$.

Proof: Please refer to Appendix D.

It is clear from (31) that the optimal choice of $\beta_{1}$ and $\beta_{2}$ reduces the gap between $P_{\mathrm{o}}$ and $P_{\mathrm{o}, \mathrm{ub}}$. Although an analytical approach for quantifying this gap is an open question and beyond the scope of this paper, we numerically show (cf. Section V.C) the difference between $P_{\mathrm{o}}$ and its upper bound when $\beta_{1}, \beta_{2}$, and the precoders are optimized according to the method to be presented in the sequel.

\footnotetext{
${ }^{7}$ The outage probabilities for OSTBC based MIMO relay system have been derived in [36] and [37] for single-antenna relay (no OSTBC at the relay) and MIMO relay with OSTBC, respectively. Although the authors of [36] and [37] consider correlated Nakagami- $m$ fading case, no precoders are employed at the source and relay nodes, and thus, there does not exist a straightforward way through which their expressions can be directly utilized to our case.
}

\section{Precoder Optimization}

The derived upper bound $P_{\mathrm{o}, \mathrm{ub}}$ is not a convex function of $\left\{\mathbf{W}_{i}, \beta_{i}\right\}, \forall i$. However, it is a convex function of $\left\{\mathbf{W}_{i}\right\}$ for fixed $\left\{\beta_{i}\right\}$, and $\left\{\beta_{i}\right\}$ for fixed $\left\{\mathbf{W}_{i}\right\}$. As a result, the precoder design problem is formulated as an alternating minimization problem where the objective is to minimize the upper bound of the OP while maintaining the energy transfer to the EH receiver above a certain threshold value $\tilde{P}^{8}$. By varying this value between $\tilde{P}_{\max }$ and $\tilde{P}_{\mathrm{ID}}$, where $\tilde{P}_{\max }$ and $\tilde{P}_{\mathrm{ID}}$ are in analogy with respect to $P_{\max }$ and $P_{\mathrm{ID}}$ defined in the perfect CSI case, we obtain the boundary of the OP-E region. The corresponding optimization problem for given $\left\{\beta_{i}^{*}\right\}$ is expressed as

$$
\begin{aligned}
& \mathcal{P}_{4}^{a}: \min _{\mathbf{W}_{i}, \forall i}\left[\mathrm{e}^{-\ln \operatorname{det}\left(\mathbf{I}_{n_{\mathrm{s}} n_{\mathrm{r}}}+\frac{\beta_{1}^{*}}{\sigma_{1}^{2}} \mathbf{\Phi}_{1}\right)+\beta_{1}^{*} t_{\mathrm{c}}}\right. \\
& \left.\quad+\mathrm{e}^{-\ln \operatorname{det}\left(\mathbf{I}_{n_{\mathrm{r}} n_{\mathrm{d}}}+\frac{\beta_{2}^{*}}{\sigma_{2}^{2}} \boldsymbol{\Phi}_{2}\right)+\beta_{2}^{*} t_{\mathrm{c}}}\right] \text { s.t } \\
& \mathrm{E}\left\{\operatorname{tr}\left(\mathbf{H}_{1, \mathrm{E}}^{H} \mathbf{H}_{1, \mathrm{E}} \mathbf{W}_{1}\right)+\operatorname{tr}\left(\mathbf{H}_{2, \mathrm{E}}^{H} \mathbf{H}_{2, \mathrm{E}} \mathbf{W}_{2}\right)\right\} \geq \frac{\tilde{\tilde{P}}}{\tilde{a}} \\
& \operatorname{tr}\left(\mathbf{W}_{1}+\mathbf{W}_{2}\right) \leq P_{\mathrm{T}}, \mathbf{W}_{1} \succeq 0, \mathbf{W}_{2} \succeq 0,
\end{aligned}
$$

whereas the optimization problem for given $\mathbf{W}_{i}^{*}\left(\boldsymbol{\Phi}_{i}^{*}\right)$, which satisfy the constraints (32b)-(32c), is given by

$$
\begin{aligned}
\mathcal{P}_{4}^{b}: \min _{\beta_{1}>0, \beta_{2}>0} f_{\mathrm{ob}} \triangleq & {\left[\mathrm{e}^{\beta_{1} t_{\mathrm{c}}} \mathrm{e}^{-\ln \operatorname{det}\left(\mathbf{I}_{n_{\mathrm{r}} n_{\mathrm{s}}}+\frac{\beta_{1}}{\sigma_{1}^{2}} \mathbf{\Phi}_{1}^{*}\right)}\right.} \\
& \left.+\mathrm{e}^{\beta_{2} t_{\mathrm{c}}} \mathrm{e}^{-\ln \operatorname{det}\left(\mathbf{I}_{n_{\mathrm{r}} n_{\mathrm{d}}}+\frac{\beta_{2}}{\sigma_{2}^{2}} \boldsymbol{\Phi}_{2}^{*}\right)}\right] .
\end{aligned}
$$

Notice that for $\mathbf{X} \in \mathcal{C}^{M \times N}$ with ZMCSCG random variables of unit variance and a deterministic matrix $\mathbf{A} \in \mathcal{C}^{M \times M}$, $\mathrm{E}\left\{\mathbf{X}^{H} \mathbf{A X}\right\}$ can be expressed as $\mathrm{E}\left\{\mathbf{X}^{H} \mathbf{A X}\right\}=\operatorname{tr}(\mathbf{A}) \mathbf{I}_{N}$ [39]. Using this fact and introducing the auxiliary variables $\tilde{\tau}_{1} \geq 0$ and $\tilde{\tau}_{2} \geq 0$, the optimization problem (32) can be expressed as

$$
\begin{aligned}
& \mathcal{P}_{4}^{a}: \min _{\mathbf{W}_{i}, \tilde{\tau}_{i}, \forall i} \mathrm{e}^{-\tilde{\tau}_{1}}+\mathrm{e}^{-\tilde{\tau}_{2}} \text { s.t. } \\
& \tilde{\tau}_{i} \leq \ln \operatorname{det}\left(\mathbf{I}_{n_{i}}+\frac{\beta_{i}^{*}}{\sigma_{i}^{2}} \mathbf{\Phi}_{i}\right)-\beta_{i}^{*} t_{\mathrm{c}}, i=1,2, \\
& \sum_{i=1}^{2} \operatorname{tr}\left(\mathbf{R}_{i, \mathrm{E}, \mathrm{r}}\right) \operatorname{tr}\left(\mathbf{R}_{i, \mathrm{E}, \mathrm{t}} \mathbf{W}_{i}\right) \geq \frac{\tilde{\tilde{P}}}{\tilde{a}},(32 \mathrm{c})
\end{aligned}
$$

where $n_{1}=n_{\mathrm{r}} n_{\mathrm{s}}$ and $n_{2}=n_{\mathrm{r}} n_{\mathrm{d}}$. The optimization problem (34) can be solved by using a convex optimization toolbox, such as CVX [32]. The solution $\left\{\beta_{i}\right\}$ of $\mathcal{P}_{4}^{b}$ can be obtained by solving $\frac{\partial f_{\mathrm{ob}}}{\partial \beta_{i}}=0$, which results into

$$
\begin{aligned}
& \frac{\partial\left[\ln \operatorname{det}\left(\mathbf{I}_{n_{i}}+\frac{\beta_{i}}{\sigma_{i}^{2}} \mathbf{\Phi}_{i}^{*}\right)\right]}{\partial \beta_{i}}=t_{\mathrm{c}} \\
& \rightarrow \sum_{n=1}^{L_{i}} \frac{\left(\lambda_{n}^{(i)}\right)^{*}}{\sigma_{i}^{2}+\beta_{i}\left(\lambda_{n}^{(i)}\right)^{*}}=t_{\mathrm{c}}, i=1,2
\end{aligned}
$$

\footnotetext{
${ }^{8}$ When this threshold value is zero, the precoder design problem turns to a scenario without an $\mathrm{EH}$ receiver and yields the best (minimum) OP.
} 
where the second equality is obtained by using the fact that $\frac{\partial \operatorname{det}(\mathbf{Y})}{\partial x}=\operatorname{det}(\mathbf{Y}) \operatorname{tr}\left\{\mathbf{Y}^{-1} \frac{\partial \mathbf{Y}}{\partial x}\right\}$. Equation (35) can be expressed as a polynomial of $L_{i}$ th degree. The positive root of (35), which yields minimum value of $f_{\mathrm{ob}}$, is the solution for $\left\{\beta_{i}\right\}$. If no such positive root is feasible, (35) can be solved using computationally efficient line search over $\left\{\beta_{i}\right\}$. It follows that the precoder optimization problem is solved by iteratively optimizing $\mathcal{P}_{4}^{a}$ and $\mathcal{P}_{4}^{b}$ until a predefined convergence accuracy is achieved. The convergence of this iterative method to a stationary point can be confirmed as follows. Notice that our problem is a two-block alternating optimization, where the blocks are $\left\{\mathbf{W}_{1}, \mathbf{W}_{2}\right\}$ and $\left\{\beta_{1}, \beta_{2}\right\}$. This means that for each iteration cycle, we only have two alternating optimization updates. Furthermore, the objective function is continuously differentiable and the feasible set is nonempty and convex. Under the observation that the modifications of $\beta_{1}>0$ and $\beta_{2}>0$ to $\beta_{1} \geq 0$ and $\beta_{2} \geq 0$, respectively, do not change the optimization problem, the feasible set is also closed. As a result, we can apply the result of Corollary 2 of [40] and verify convergence of the alternating optimization to a stationary point. However, due to the fact that the objective function cannot be shown to be convex or belong to a group of generalized convex functions, it cannot be guaranteed that the iterative approach converges to the global minimum. Nonetheless, in the considered numerical examples (cf. Section V), we find that the alternating minimization technique gives (almost) the same result as the one obtained by solving $\mathcal{P}_{4}^{a}$ for a fine grid of $\left\{\beta_{i}\right\}$. Moreover, it is interesting to note that the search over $\left\{\beta_{i}\right\}$ can be limited to a very small region due to the fact that $\beta_{i}$ can be upper bounded as $\beta_{i} \leq \frac{L_{i}}{t_{c}}$. This upper bound is obvious from (35) which is a necessary condition for the joint optimal solutions of $\left(\mathbf{W}_{i}, \beta_{i}, \forall i\right)$.

- Remark 5: In contrast to the optimization problem in the perfect CSI case, the optimal $\left\{\mathbf{W}_{i}^{*}\right\}$ are likely to have higher ranks due to the fact that $\left\{\tilde{\tau}_{i}\right\}$ are related to $\left\{\mathbf{W}_{i}\right\}$ through the log-determinant function. As a result, in general $\mathcal{P}_{4}^{a}$ is a relaxed optimization problem. However, when Alamouti code is used to avoid the rate loss, the solutions of $\mathcal{P}_{4}^{a}$ are optimal for $n_{\mathrm{r}}=n_{\mathrm{s}}=2$. For $n_{\mathrm{r}}=n_{\mathrm{s}}>2$, we can employ rank-one or rank-two approximation methods, such as randomization [30] (see also references therein), to generate a feasible solution from $\mathcal{P}_{4}^{a}$.

- Remark 6: We describe an alternative to the OP-E region corresponding to $\mathcal{P}_{4}$, which is based on the maximization of the LTA-SNR. Notice that this approach is inspired from other applications, such as power control [41] and beamforming [42], [43], where the relation between the LTA-SNR and OP is reported, i.e., maximizing LTA-SNR is shown to be approximately equivalent to minimizing the OP ${ }^{9}$. Following the approach of [41], the LTA-SNR is defined as $f_{\mathrm{av}}=\frac{\bar{\gamma}_{1} \bar{\gamma}_{2}}{\bar{\gamma}_{1}+\bar{\gamma}_{2}+1}$, where $\bar{\gamma}_{1}=\mathrm{E}\left\{\gamma_{1}\right\}$ and $\bar{\gamma}_{2}=\mathrm{E}\left\{\gamma_{2}\right\}$. The LTA-SNR based optimization problem is then given by

$$
\begin{aligned}
& \mathcal{P}_{5}: \max _{\mathbf{W}_{1}, \mathbf{W}_{2}, \bar{\gamma}_{1}, \bar{\gamma}_{2}} \frac{\bar{\gamma}_{1} \bar{\gamma}_{2}}{\bar{\gamma}_{1}+\bar{\gamma}_{2}+1} \text { s.t } \\
& \operatorname{tr}\left(\mathbf{R}_{i, \mathrm{I}, \mathrm{r}}\right) \operatorname{tr}\left(\mathbf{R}_{i, \mathrm{I}, \mathrm{t}} \mathbf{W}_{1}\right) \geq \bar{\gamma}_{i} \sigma_{i}^{2}, \forall i,
\end{aligned}
$$

\footnotetext{
${ }^{9}$ The authors in [41] use a different notion such as certainty equivalent margin (CEM), however, its close relation to LTA-SNR is obvious.
}

where (36b) is due to the fact that $\mathrm{E}\left\{\mathbf{X}^{H} \mathbf{A X}\right\}=\operatorname{tr}(\mathbf{A}) \mathbf{I}_{N}$ [39] for $\mathbf{X}$ with ZMCSCG random variables of unit variance. Notice that the structure of problem $\mathcal{P}_{5}$ is similar to the problem (18) or (19). The only difference between these problems is that (18) depends on the instantaneous channels, whereas $\mathcal{P}_{5}$ depends on channel covariance matrices. As a result, the results of the Propositions 2 and 3 are also applicable to the problem $\mathcal{P}_{5}$. Consequently, $\mathcal{P}_{5}$ can be solved as $\mathcal{P}_{3}$ and the corresponding rank-one optimal solutions of $\mathbf{W}_{1}$ and $\mathbf{W}_{2}$ can be always obtained.

\section{NUMERICAL RESULTS}

In this section, we present numerical results that characterize the tradeoffs between rate and energy, and OP and energy. For all simulation examples, we take $\sigma_{1}^{2}=\sigma_{2}^{2}=\sigma^{2}, n_{\mathrm{s}}=n_{\mathrm{r}}=$ $2, \mu=1$, and consider that both the source and relay use the Alamouti code $\left(R_{\mathrm{c}}=1\right)$. For a uniform linear array with a halfwavelength spacing at each MIMO node, the spatial correlation matrices are given by [44]

$$
\begin{aligned}
{\left[\mathbf{R}_{i, \mathrm{I} / \mathrm{E}, \mathrm{r} / \mathrm{t}}\right]_{m, n}=} & \mathrm{e}^{\mathrm{j} \pi(m-n) \sin \theta_{i, \mathrm{I} / \mathrm{E}, \mathrm{r} / \mathrm{t}}} \\
& \times \mathrm{e}^{-\left(\pi(m-n) \sigma_{\theta}^{i, \mathrm{I} / \mathrm{E}, \mathrm{r} / \mathrm{t}} \cos \theta_{i}^{\mathrm{I} / \mathrm{E}, \mathrm{r} / \mathrm{t}}\right)^{2} / 2}
\end{aligned}
$$

where the notation xi/yi stands for either index xi or yi and [ $]_{m, n}$ represents the $m$ th row and $n$th column of $\mathbf{R}_{i, \mathrm{I} / \mathrm{E}, \mathrm{r} / \mathrm{t}}$. Notice that $\theta_{1, \mathrm{I}, \mathrm{t}} / \theta_{1, \mathrm{I}, \mathrm{r}}$ is the central angle of the outgoing/incoming rays from the source/to the relay and $\sigma_{\theta}^{1, \mathrm{I}, \mathrm{r}} / \sigma_{\theta}^{1, \mathrm{I}, \mathrm{t}}$ is the standard deviation of the corresponding angular spread. Similar

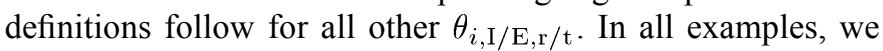
take $\sigma_{\theta}^{i, \mathrm{I} / \mathrm{E}, \mathrm{r} / \mathrm{t}}=\sigma_{\theta}$ for all $i$, and

$$
\begin{aligned}
\theta_{1, \mathrm{I}, \mathrm{t}} & =2^{\circ}, \theta_{1, \mathrm{I}, \mathrm{r}}=4^{\circ}, \theta_{2, \mathrm{I}, \mathrm{t}}=6^{\circ}, \theta_{2, \mathrm{I}, \mathrm{r}}=8^{\circ}, \\
\theta_{1, \mathrm{E}, \mathrm{t}} & =30^{\circ}, \theta_{1, \mathrm{E}, \mathrm{r}}=35^{\circ}, \theta_{2, \mathrm{E}, \mathrm{t}}=40^{\circ}, \theta_{2, \mathrm{E}, \mathrm{r}}=45^{\circ} .
\end{aligned}
$$

The average SNR for the S-R and R-ID links is defined as $\gamma_{\mathrm{av}} \triangleq \frac{P_{\mathrm{T}}}{\sigma^{2}}$, where $\gamma_{\mathrm{av}}$ is varied by changing $P_{\mathrm{T}}$ and taking $\sigma^{2}=1$. The optimization problems $\mathcal{P}_{3}, \mathcal{P}_{4}^{a}$ and $\mathcal{P}_{5}$ are solved using the CVX software [32]. While solving $\mathcal{P}_{3}$ (for each channel realization) and $\mathcal{P}_{5}$, we verified that the condition $\frac{e_{i, \mathrm{v}, 2}}{e_{i, \mathrm{v}}}<10^{-4}$ holds true, where $e_{i, \mathrm{v}, 1}$ and $e_{i, \mathrm{v}, 2}$ are respectively, the largest and second largest eigenvalues of the optimal solutions of $\left\{\mathbf{W}_{i}\right\}_{i=1}^{2}$ obtained from the CVX toolbox. This indicates that optimal solutions of $\left\{\mathbf{W}_{i}\right\}_{i=1}^{2}$ are roughly rank-one matrices.

\section{A. Effect of Correlation on R-E Region}

The effect of spatial correlation on the boundary of R-E region is shown in Fig. 2. The performance of both the uncorrelated case, where all channel correlation matrices are identity matrices, and the correlated case, where the channel correlation matrices as given by (37), are considered. The boundaries of the R-E regions are obtained by averaging over 200 independent realizations of $\left\{\mathbf{H}_{i, \mathrm{I}, \mathrm{w}}\right\}_{i=1}^{2}$ and $\left\{\mathbf{H}_{i, \mathrm{E}, \mathrm{w}}\right\}_{i=1}^{2}{ }^{10}$. We take $\gamma_{\mathrm{av}}=10$ $\mathrm{dB}$ and $n_{\mathrm{d}}=n_{\mathrm{e}}=4$. It can be observed from this figure that energy transfer improves when correlation increases, i.e., when $\sigma_{\theta}$ decreases. However, the corresponding information transfer

\footnotetext{
${ }^{10}$ The elements of these matrices are drawn from ZMCSCG distribution.
} 


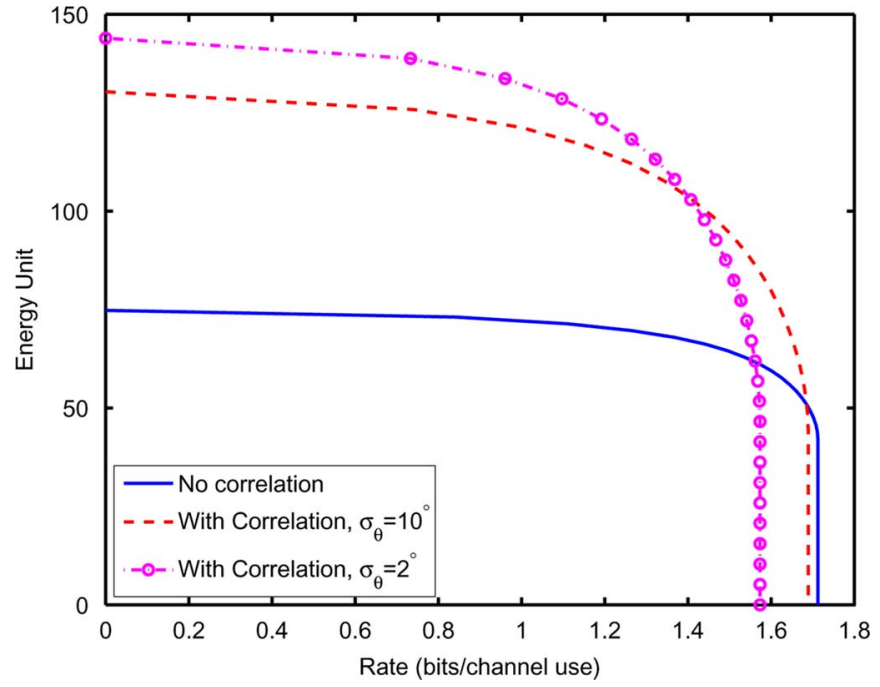

Fig. 2. R-E regions with uncorrelated and correlated channels for $\gamma_{\mathrm{av}}=10 \mathrm{~dB}$

reduces. This result can be explained as follows. Notice that higher spatial correlation means larger values for the maximum eigenvalues of the channel covariance matrices. Based on optimal solutions of (12), the average $P_{\max }$ turns to be linearly proportional to the largest eigenvalues of $\mathrm{E}\left\{\mathbf{H}_{i, \mathrm{E}}^{H} \mathbf{H}_{i, \mathrm{E}}\right\}, \forall i$, meaning that correlation increases average maximum energy transfer. On the other hand, instantaneous information rate is limited by the weaker single-hop link, i.e., the link with smaller largest eigenvalue. It is known that in a double-sided correlated point-to-point MIMO channel with rank-one optimal precoder, higher spatial correlation also results into higher spreading of the instantaneous largest eigenvalue [45], i.e., probability of having lower values of the largest eigenvalue also increases. Linking above two facts, it can be intuitively said that an increase in spatial correlation can cause reduction in average $R_{\max }$.

\section{B. R-E Region}

In Figs. 3 and 4, we plot the boundary of the R-E region. The R-E boundaries are obtained by averaging over 200 independent realizations of $\left\{\mathbf{H}_{i, \mathrm{I}, \mathrm{w}}\right\}_{i=1}^{2}$ and $\left\{\mathbf{H}_{i, \mathrm{E}, \mathrm{w}}\right\}_{i=1}^{2}$. The spatial correlation matrices are taken as identity matrices (uncorrelated case). Fig. 3 shows the tradeoff between the maximum energy harvested by the $\mathrm{EH}$ receiver and the maximum information rate transferred to the ID receiver for different values of $n_{\mathrm{d}}$ with $n_{\mathrm{e}}=2$ and $\gamma_{\mathrm{av}}=20 \mathrm{~dB}$. It can be observed from this figure that information transfer increases when $n_{\mathrm{d}}$ increases, whereas the energy transfer remains the same. In Fig. 4, the R-E region is displayed for different values of $n_{\mathrm{e}}$ by keeping $n_{\mathrm{d}}=4$ and $\gamma_{\mathrm{av}}=20 \mathrm{~dB}$. It can be observed from this figure that the energy transfer increases with the increasing value of $n_{\mathrm{e}}$, whereas the information transfer remains the same.

\section{OP-E Region}

For all simulation results of this subsection, we take $r_{0}=1$ bit/channel use and set $\left|\beta_{i}^{m+1}-\beta_{i}^{m}\right| \leq 10^{-3}$ and $\| \mathbf{W}_{i}^{m+1}-$ $\mathbf{W}_{i}^{m} \| \leq 10^{-4}$, where $m$ stands for iteration step, for stopping (convergence criterion) the iterative optimization $\mathcal{P}_{4}$.

In Fig. 5, we compare the exact OP (evaluated by (29)) and simulated OP for different $n_{\mathrm{d}}$ and $n_{\mathrm{e}}=4$. The upper bound

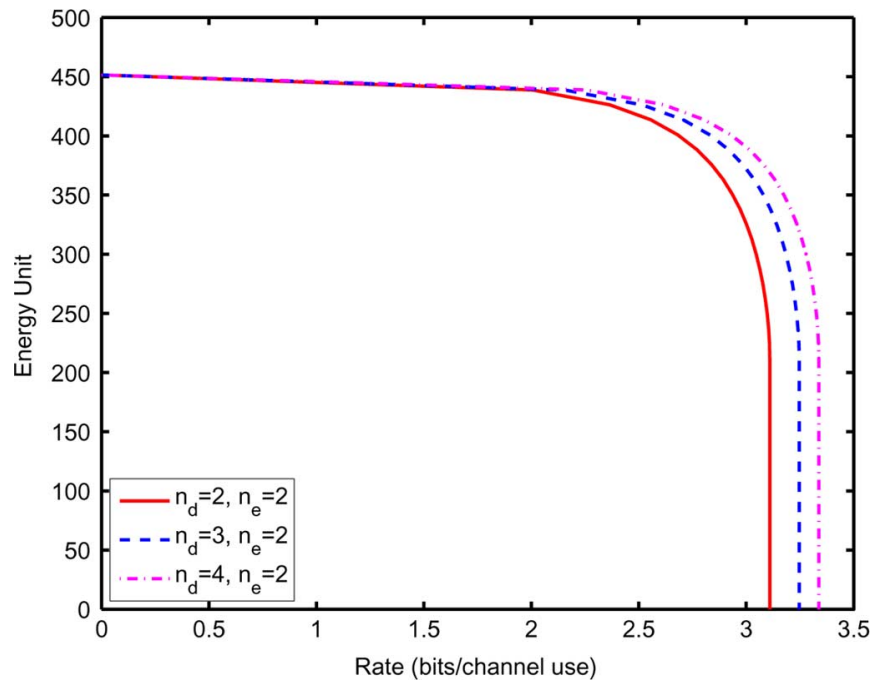

Fig. 3. R-E regions for $\gamma_{\mathrm{av}}=20 \mathrm{~dB}$ and $n_{\mathrm{e}}=2$.

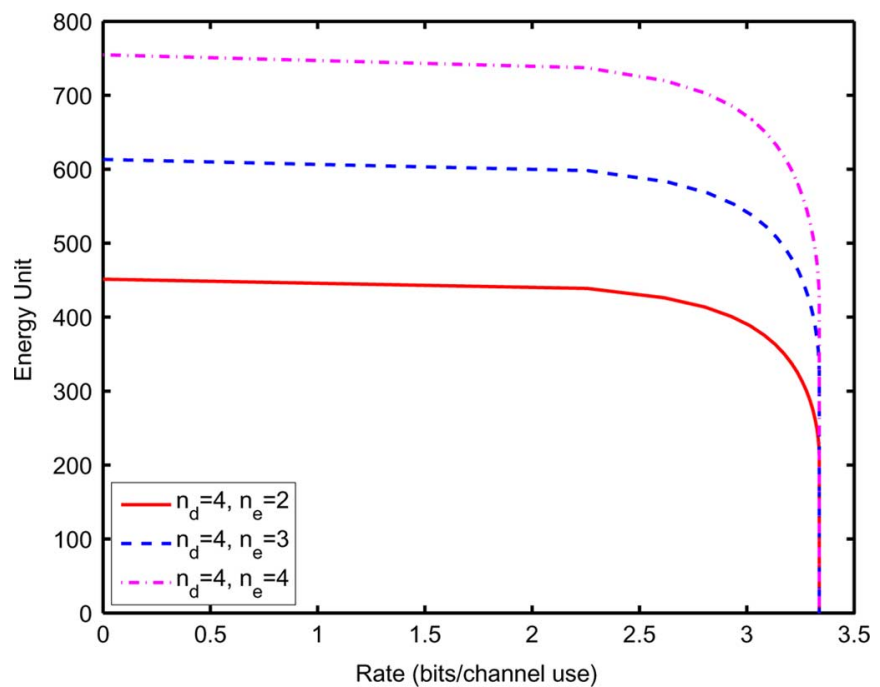

Fig. 4. R-E regions for $\gamma_{\mathrm{av}}=20 \mathrm{~dB}$ and $n_{\mathrm{d}}=4$.

of the OP is also displayed in this figure by taking $\nu=1$ and $\sigma_{\theta}=5^{\circ}$. Notice that $\nu=1$ is taken since this yields smaller gap between $P_{\mathrm{o}}$ and its upper bound without affecting $P_{\mathrm{o}}$. This shows that, although factor $\frac{1}{2}$ is required in (54) to strictly upper bound $\tilde{\gamma}$, due to the fact that the bound (54) is tight at high SNR region without this factor, better values of $P_{\mathrm{o}, \mathrm{ub}}$ are obtained in the considered simulation example. The precoders are obtained by iteratively solving $\mathcal{P}_{4}^{a}$ (with no power constraint for $\mathrm{EH}$ receiver, i.e., $\tilde{P}=0$ ) and $\mathcal{P}_{4}^{b}$. It can be observed from this figure that the simulated and analytical OP exhibit very fine matching for both $n_{\mathrm{d}}=2$ and $n_{\mathrm{d}}=4$.

Fig. 6 shows the tradeoff between the energy harvested by the $\mathrm{EH}$ receiver and the OP maintained to the ID receiver for different values of $\sigma_{\theta}$, when $n_{\mathrm{d}}=n_{\mathrm{e}}=4$ and $\gamma_{\mathrm{av}}=15 \mathrm{~dB}$. The OP-E boundary obtained with the LTA-SNR maximization approach is also shown in Fig. 6. It can be observed from this figure that energy transfer increases when $\sigma_{\theta}$ decreases, i.e., correlation increases. The corresponding OP also increases. This observation can be explained as follows. The asymptotic OP (with respect to $\gamma_{\mathrm{av}}$ ) is known to provide the diversity gain 


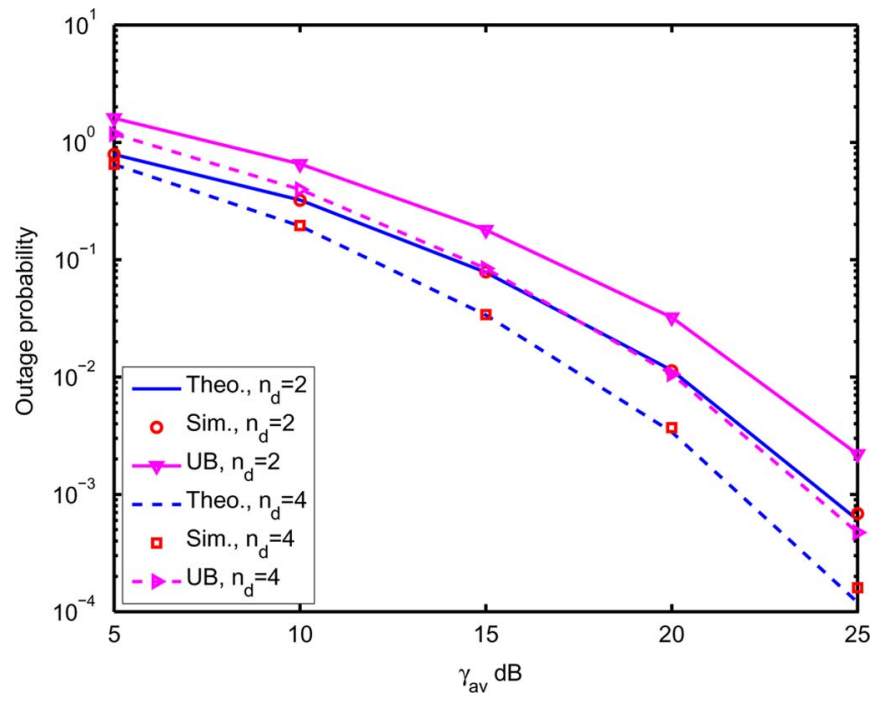

Fig. 5. Comparison of theoretical and simulated OP and its upper bound for $n_{\mathrm{e}}=4$.

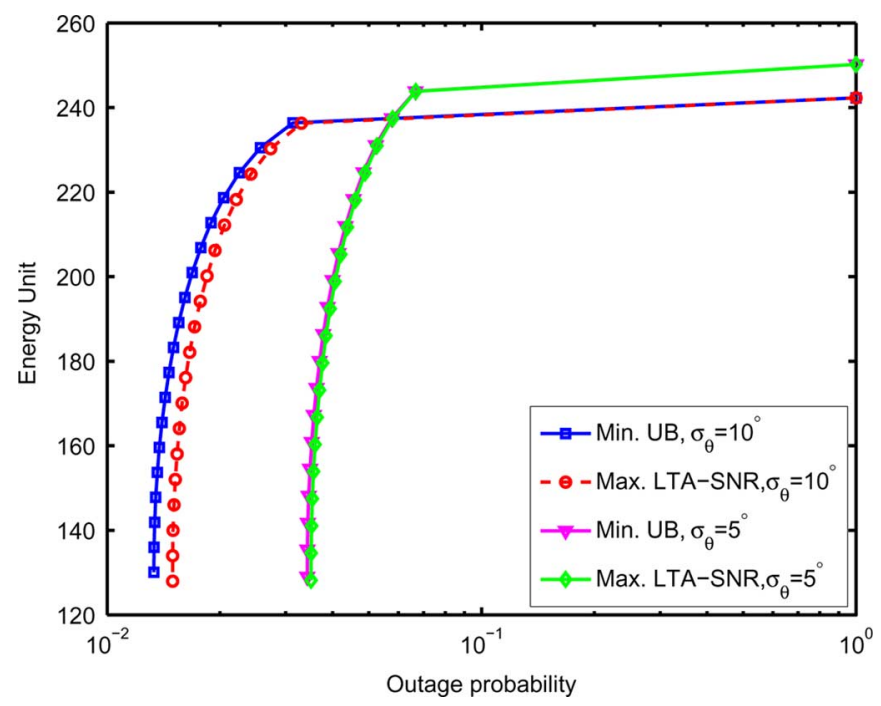

Fig. 6. OP-E regions for $\gamma_{\mathrm{av}}=15 \mathrm{~dB}$ and $n_{\mathrm{d}}=n_{\mathrm{e}}=4$.

of the system. With a simple manipulation of $P_{\mathrm{o}, \mathrm{ub}}$ for high SNR (e.g., $\sigma^{2} \rightarrow 0$ with a given $P_{\mathrm{T}}$ ), it can be readily shown that the diversity gain for the $i$ th hop is equal to $\operatorname{rank}\left(\boldsymbol{\Phi}_{i}\right)$. This means that the diversity gain for the relay system is $\min \left(\operatorname{rank}\left(\boldsymbol{\Phi}_{1}\right), \operatorname{rank}\left(\boldsymbol{\Phi}_{2}\right)\right)$. For a given optimal solution of $\mathbf{W}_{i}, \operatorname{rank}\left(\boldsymbol{\Phi}_{i}\right)$ decreases when $\operatorname{rank}\left(\mathbf{R}_{i, \mathrm{I}}\right)$ decreases, which happens when spatial correlation increases. Similar result w.r.t. symbol error probability has been reported in a point-to-point MIMO system [24]. Furthermore, in Fig. 6, the proposed upper bound minimization approach provides better performance than the LTA-SNR maximization in terms of the OP-E region. The OP-E tradeoff for different values of $n_{\mathrm{d}}$ is shown in Fig. 7 for both the upper bound minimization and LTA-SNR maximization methods. In this figure, we take $n_{\mathrm{e}}=4, \sigma_{\theta}=10^{\circ}$ and $\gamma_{\mathrm{av}}=20 \mathrm{~dB}$. By comparing Fig. 7 with Fig. 6 , it can be observed that the proposed method significantly outperforms LTA-SNR, especially when $\gamma_{\mathrm{av}}$ and $n_{\mathrm{d}}$ increase. For a given antenna configuration and $\sigma_{\theta}$, increase in $\gamma_{\mathrm{av}}$ improves both

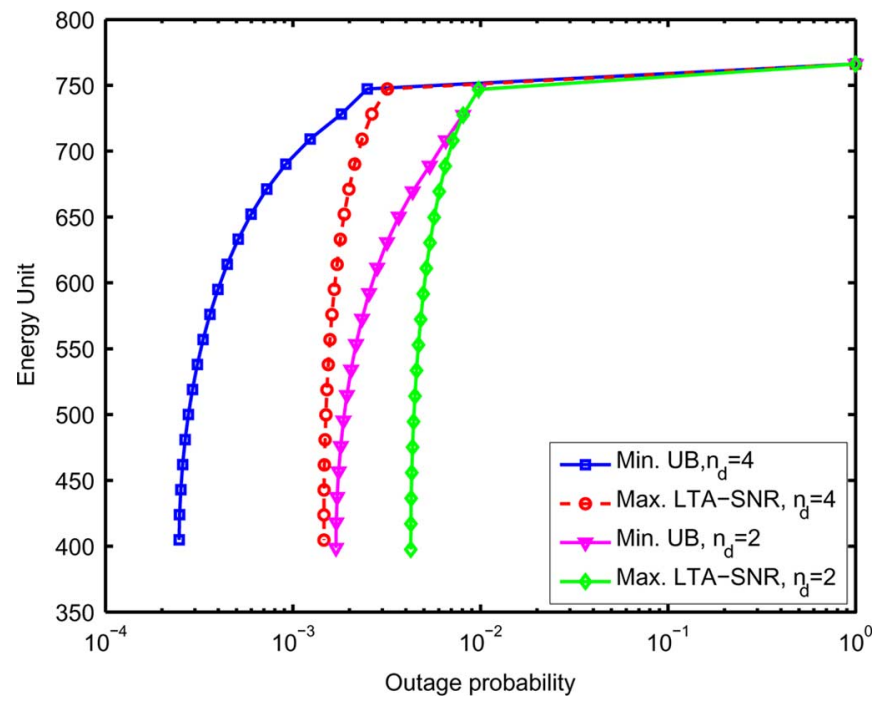

Fig. 7. OP-E regions for $\gamma_{\mathrm{av}}=20 \mathrm{~dB}$ and $n_{\mathrm{e}}=4$.

OP and energy transfer, whereas, for the given $\gamma_{\mathrm{av}}$ and $n_{\mathrm{e}}$, the OP improves with the increasing $n_{\mathrm{d}}$ and/or $\sigma_{\theta}$.

\section{CONCLUSION}

We proposed optimum performance boundaries of a dual-hop OSTBC-based MIMO relay system in the presence of a lowpowered energy harvesting receiver. The tradeoffs between information rate and energy and between outage probability and energy were characterized by the boundaries of the R-E and OP-E regions, respectively. When perfect CSI is available, the proposed joint source and relay precoder design is shown to be optimal and the Alamouti codes can be employed for an arbitrary number of antennas at the transmit nodes and support up to seven simultaneously existing EH receivers. For the case where only the second-order statistics of CSI is known, the performance tradeoffs are characterized by the OP-E region. In this case, a tractable precoder optimization problem based on the upper bound of the OP is proposed. Numerical results show that the proposed method provides better tradeoff between OP and energy when compared to the method based on maximizing the LTA-SNR. The role of the numbers of antennas and average SNR on the boundaries of the R-E and OP-E regions is demonstrated with numerical results. It is observed that spatial correlation increases energy transfer for both cases with instantaneous and second-order statistics of CSI, while the corresponding information transfer/outage probability reduces/increases.

\section{APPENDIX A}

ProOF OF PROPOSITION 2

Let $\left(\mathbf{W}_{1}^{*}, \mathbf{W}_{2}^{*}, \gamma_{1}^{*}, \gamma_{2}^{*}\right)$ be an optimal solution of the problem (18). Consider the following problem

$$
\begin{aligned}
& \min _{\mathbf{W}_{1}, \mathbf{W}_{2}} \operatorname{tr}\left(\mathbf{W}_{1}+\mathbf{W}_{2}\right) \text { s.t. } \\
& \operatorname{tr}\left(\mathbf{H}_{1, \mathrm{I}}^{H} \mathbf{H}_{1, \mathrm{I}} \mathbf{W}_{1}\right) \geq \gamma_{1}^{*} \sigma_{1}^{2}, \operatorname{tr}\left(\mathbf{H}_{2, \mathrm{I}}^{H} \mathbf{H}_{2, \mathrm{I}} \mathbf{W}_{2}\right) \geq \gamma_{2}^{*} \sigma_{2}^{2},
\end{aligned}
$$




$$
\begin{aligned}
& \operatorname{tr}\left(\mathbf{H}_{1, \mathrm{E}}^{H} \mathbf{H}_{1, \mathrm{E}} \mathbf{W}_{1}\right)+\operatorname{tr}\left(\mathbf{H}_{2, \mathrm{E}}^{H} \mathbf{H}_{2, \mathrm{E}} \mathbf{W}_{2}\right) \geq \frac{\bar{P}}{\tilde{a}}, \\
& \mathbf{W}_{1} \succeq \mathbf{0}, \mathbf{W}_{2} \succeq \mathbf{0}
\end{aligned}
$$

Let $\left(\hat{\mathbf{W}}_{1}, \hat{\mathbf{W}}_{2}\right)$ be an optimal solution of problem (38). We claim that $\left(\hat{\mathbf{W}}_{1}, \hat{\mathbf{W}}_{2}, \gamma_{1}^{*}, \gamma_{2}^{*}\right)$ is also an optimal solution of problem (18). The proof is as follows. Since $\left(\mathbf{W}_{1}^{*}, \mathbf{W}_{2}^{*}\right)$ is feasible to (18), it can be verified that $\left(\mathbf{W}_{1}^{*}, \mathbf{W}_{2}^{*}\right)$ is also feasible to (38). As such, we have

$$
\operatorname{tr}\left(\hat{\mathbf{W}}_{1}+\hat{\mathbf{W}}_{2}\right) \leq \operatorname{tr}\left(\mathbf{W}_{1}^{*}+\mathbf{W}_{2}^{*}\right) \leq P_{T} .
$$

The above equation implies that $\left(\hat{\mathbf{W}}_{1}, \hat{\mathbf{W}}_{2}, \gamma_{1}^{*}, \gamma_{2}^{*}\right)$ is feasible to (18). In addition, since $\left(\hat{\mathbf{W}}_{1}, \hat{\mathbf{W}}_{2}, \gamma_{1}^{*}, \gamma_{2}^{*}\right)$ and $\left(\mathbf{W}_{1}^{*}, \mathbf{W}_{2}^{*}, \gamma_{1}^{*}, \gamma_{2}^{*}\right)$ attain the same objective value in (18), $\left(\hat{\mathbf{W}}_{1}, \hat{\mathbf{W}}_{2}, \gamma_{1}^{*}, \gamma_{2}^{*}\right)$ is indeed optimal to (18).

Next, we show that optimal solutions with $\operatorname{rank}\left(\hat{\mathbf{W}}_{1}\right)=$ $\operatorname{rank}\left(\hat{\mathbf{W}}_{2}\right)=1$ can always be obtained in (38). First, by applying the Shapiro-Barvinok-Pataki (SBP) rank reduction theorem for complex-valued SDPs [[31], Lemma 3.1] (also [30] for a friendly description), we know that there is an optimal solution $\left(\hat{\mathbf{W}}_{1}, \hat{\mathbf{W}}_{2}\right)$ of (38) such that

$$
\left(\operatorname{rank}\left(\hat{\mathbf{W}}_{1}\right)\right)^{2}+\left(\operatorname{rank}\left(\hat{\mathbf{W}}_{2}\right)\right)^{2} \leq 3 .
$$

Consequently, we have $\operatorname{rank}\left(\hat{\mathbf{W}}_{1}\right) \leq 1$ and $\operatorname{rank}\left(\hat{\mathbf{W}}_{2}\right) \leq 1$. Second, as a necessary condition, we must have $\operatorname{rank}\left(\hat{\mathbf{W}}_{1}\right) \geq$ 1 and $\operatorname{rank}\left(\hat{\mathbf{W}}_{2}\right) \geq 1$. This can be shown by contradiction. Suppose that $\operatorname{rank}\left(\hat{\mathbf{W}}_{1}\right)=0$, or, equivalently, $\hat{\mathbf{W}}_{1}=\mathbf{0}$. Then, to satisfy (38b), we must have $\gamma_{1}^{*}=0$ (except for the trivial case of $\mathbf{H}_{1, \mathrm{I}}=\mathbf{0}$ ). However, having $\gamma_{1}^{*}=0$ is equivalent to zero rate in (18), which is not optimal (again, except for the trivial case of $\mathbf{H}_{1, \mathrm{I}}=\mathbf{0}$ or $\mathbf{H}_{2, \mathrm{I}}=\mathbf{0}$ ). Combining the two results, we conclude that $\operatorname{rank}\left(\hat{\mathbf{W}}_{1}\right)=\operatorname{rank}\left(\hat{\mathbf{W}}_{2}\right)=1$. This completes the proof of Proposition 2.

\section{APPENDIX B}

\section{PROOF OF PROPOSITION 3}

By introducing positive real auxiliary variables $\tau_{1}, \tau_{2}$ and $\tilde{t}$, we can express the optimization problem (19) as

$$
\begin{aligned}
& \min _{\left\{\left\{\mathbf{W}_{i}, \gamma_{i}, \tau_{i}, \forall i\right\}, \tilde{t}\right\}} \tau_{1}+\tau_{2}+\tilde{t} \text { s.t. } \\
& \tilde{t} \gamma_{1} \gamma_{2} \geq 1, \\
& \tau_{i} \gamma_{i} \geq 1, \forall i, \quad(18 \mathrm{~b})-(18 \mathrm{~d}) .
\end{aligned}
$$

We introduce further auxiliary variables $u_{1} \geq 0$ and $u_{2} \geq 0$, and rewrite (41b) as

$$
\tilde{t} \gamma_{1} \gamma_{2} \geq 1 \longleftrightarrow u_{1}^{2} u_{2}^{2} \geq 1, u_{1}^{2}=\tilde{t} \gamma_{1}, u_{2}^{2}=\gamma_{2}
$$

In order to proceed to final formulation, we employ the result of the following lemma.

Lemma 1: For the optimization problem (41), the constraint (42) can be equivalently expressed as

$$
\tilde{t} \gamma_{1} \gamma_{2} \geq 1 \longleftrightarrow u_{1} u_{2} \geq 1, u_{1}^{2} \leq \tilde{t} \gamma_{1}, u_{2}^{2} \leq \gamma_{2}
$$

Proof: We prove this Lemma by the method of contradiction. Let us assume that the constraints $u_{1}^{2} \leq \tilde{t} \gamma_{1}$ and $u_{2}^{2} \leq \gamma_{2}$ are inactive at the optimality, i.e., $u_{1}^{2}<\tilde{t} \gamma_{1}$ and $u_{2}^{2}<\gamma_{2}$. We can scale $u_{1}$ and $u_{2}$ by positive scalars $\eta_{1}$ and $\eta_{2}$ such that the inequality constraints $u_{1}^{2}<\tilde{t} \gamma_{1}$ and $u_{2}^{2}<\gamma_{2}$ become active, i.e., we have $\eta_{1}^{2} u_{1}^{2}=\tilde{t} \gamma_{1}$ and $\eta_{2}^{2} u_{2}^{2}=\gamma_{2}$. This does not affect the other constraints of (41) including the constraint $u_{1}^{2} u_{2}^{2} \geq 1$. The corresponding achieved objective function in this case turns to

$$
\tilde{f}_{\mathrm{ob}}=\frac{1}{\gamma_{1}}+\frac{\gamma_{1}+\eta_{1}^{2} u_{1}^{2} \eta_{2}^{2} u_{2}^{2}}{\gamma_{1} \gamma_{2}} \geq \frac{1}{\gamma_{1}}+\frac{\gamma_{1}+1}{\gamma_{1} \gamma_{2}},
$$

because $\eta_{1}^{2} u_{1}^{2} \eta_{2}^{2} u_{2}^{2} \geq 1$ due to the facts that $u_{1} u_{2} \geq 1, \eta_{1} \geq 1$ and $\eta_{2} \geq 1$. This shows that higher objective function value will be achieved with $u_{1}^{2}<\tilde{t} \gamma_{1}$ and $u_{2}^{2}<\gamma_{2}$. This contradicts that (44) is minimum. Therefore, the constraints $u_{1}^{2} \leq \tilde{t} \gamma_{1}, u_{2}^{2} \leq \gamma_{2}$ are not inactive at the optimality. It follows that the proof of Lemma 1 is complete.

Using Schur-complement theorem [28], the constraints $u_{1}^{2} \leq$ $\tilde{t} \gamma_{1}$ and $u_{2}^{2} \leq \gamma_{2}$ can be equivalently expressed as

$$
\begin{aligned}
& u_{1}^{2} \leq \tilde{t} \gamma_{1} \longmapsto\left[\begin{array}{cc}
\tilde{t} & u_{1} \\
u_{1} & \gamma_{1}
\end{array}\right] \succeq 0, \\
& u_{2}^{2} \leq \gamma_{2} \longmapsto\left[\begin{array}{cc}
1 & u_{2} \\
u_{2} & \gamma_{2}
\end{array}\right] \succeq 0,
\end{aligned}
$$

whereas the hyperbolic constraints $u_{1} u_{2} \geq 1$ and $\tau_{i} \gamma_{i} \geq 1, \forall i$ can be, respectively, expressed as

$$
\begin{aligned}
u_{1}+u_{2} & \geq\left\|\left[\sqrt{2}, u_{1}, u_{2}\right]^{T}\right\|, \\
\tau_{i}+\gamma_{i} & \geq\left\|\left[\sqrt{2}, \tau_{i}, \gamma_{i}\right]^{T}\right\|, \forall i .
\end{aligned}
$$

Thus, the proof of Proposition 3 is completed.

\section{APPENDIX C \\ ProOF OF Proposition 4}

We have considered that $\left\{\lambda_{i}^{(1)}\right\}_{i=1}^{L_{1}}$ are distinct for all $i$ and $\left\{\lambda_{k}^{(2)}\right\}_{k=1}^{L_{2}}$ are distinct for all $k$. The following lemma provides the probability density function (PDF) of $\alpha_{1}$ and $\alpha_{2}$.

Lemma 2 (p. 11, [46]): The PDFs of the random variables $\alpha_{1}$ and $\alpha_{2}$ are, respectively, given by

$$
f_{\alpha_{1}}(z)=\sum_{i=1}^{L_{1}} a_{i} \mathrm{e}^{-\frac{z}{\lambda_{i}^{(1)}}}, f_{\alpha_{2}}(z)=\sum_{k=1}^{L_{2}} b_{k} \mathrm{e}^{-\frac{z}{\lambda_{k}^{(2)}}}
$$

where $z \geq 0$, and for $L_{1}>1$ and $L_{2}>1$

$$
a_{i}=\frac{\left(\lambda_{i}^{(1)}\right)^{L_{1}-2}}{\prod_{j=1, j \neq i}^{L_{1}} \lambda_{i}^{(1)}-\lambda_{j}^{(1)}}, b_{k}=\frac{\left(\lambda_{k}^{(2)}\right)^{L_{2}-2}}{\prod_{l=1, l \neq k}^{L_{2}} \lambda_{k}^{(2)}-\lambda_{l}^{(2)}} .
$$

For $L_{1}=1$ and $L_{2}=1, a_{i}$ and $b_{k}$ takes the values of $\frac{1}{\lambda_{i}^{(1)}}$ and $\frac{1}{\lambda_{k}^{(2)}}$, respectively.

${ }^{k}$ Since $\gamma_{1}=\frac{\alpha_{1}}{\sigma_{1}^{2}}$ and $\gamma_{2}=\frac{\alpha_{2}}{\sigma_{2}^{2}}$, the PDFs of $\gamma_{1}$ and $\gamma_{2}$ are readily given as

$$
f_{\gamma_{1}}(z)=\sigma_{1}^{2} f_{\alpha_{1}}\left(z \sigma_{1}^{2}\right), f_{\gamma_{2}}(z)=\sigma_{2}^{2} f_{\alpha_{2}}\left(z \sigma_{2}^{2}\right) .
$$


Applying (49), the OP can be expressed as

$$
\begin{aligned}
P_{\mathrm{o}} & =\operatorname{Pr}\left\{\frac{\gamma_{1} \gamma_{2}}{\gamma_{1}+\gamma_{2}+1} \leq \gamma_{0}\right\} \\
& =\int_{0}^{\infty} \operatorname{Pr}\left\{\gamma_{1}\left(x-\gamma_{0}\right) \leq \gamma_{0}(x+1)\right\} f_{\gamma_{2}}(x) d x \\
& =\int_{0}^{\gamma_{0}} f_{\gamma_{2}}(x) d x+\int_{\gamma_{0}}^{\infty} \operatorname{Pr}\left\{\gamma_{1} \leq \frac{\gamma_{0}(x+1)}{x-\gamma_{0}}\right\} f_{\gamma_{2}}(x) d x
\end{aligned}
$$

where the third equality is the result of separating the integral into two parts and using the fact that $\operatorname{Pr}\left\{\gamma_{1}\left(x-\gamma_{0}\right) \leq \gamma_{0}(x+\right.$ $1)\}=1$ for $0 \leq x<\gamma_{0}$. Substituting (47)-(49) into (50), noting that $\sum_{i=1}^{L_{1}} a_{i} \lambda_{i}^{(1)}=1$, and making a variable substitution $x^{\prime}=x-\gamma_{0}$, we obtain

$$
\begin{aligned}
P_{o}=1-\sigma_{2}^{2} \sum_{i=1}^{L_{1}} \sum_{k=1}^{L_{2}} a_{i} \lambda_{i}^{(1)} b_{k} \mathrm{e}^{-\frac{\gamma_{0} \sigma_{1}^{2}}{\lambda_{i}^{(1)}-\frac{\gamma_{0} \sigma_{2}^{2}}{\lambda_{k}^{(2)}}}} \\
\times \int_{0}^{\infty} \mathrm{e}^{-\frac{\gamma_{0}\left(\gamma_{0}+1\right) \sigma_{1}^{2}}{\lambda_{i}^{(1)} x^{\prime}}-\frac{x^{\prime} \sigma_{2}^{2}}{\lambda_{k}^{(2)}}} d x^{\prime} .
\end{aligned}
$$

Applying the following result [(3.324.1), [47]]

$$
\int_{0}^{\infty} \mathrm{e}^{-\frac{\beta}{4 x^{\prime}}-\gamma x^{\prime}} d x^{\prime}=\sqrt{\frac{\beta}{\gamma}} K_{1}(\sqrt{\beta \gamma}),
$$

expression (29) is obtained. This completes the proof of Proposition 4.

\section{APPENDIX D}

\section{PROOF OF PROPOSITION 5}

We first find a lower bound on $\tilde{\gamma}=\gamma+1=1+\frac{\gamma_{1} \gamma_{2}}{\gamma_{1}+\gamma_{2}+1}$. Notice that

$$
\tilde{\gamma}=\frac{1}{\frac{\gamma_{1}+\gamma_{2}+1}{\left(1+\gamma_{1}\right)\left(1+\gamma_{2}\right)}} \geq \frac{1}{\frac{1}{\gamma_{1}+1}+\frac{1}{\gamma_{2}+1}}
$$

which is a tight bound when $\frac{1}{\left(1+\gamma_{1}\right)\left(1+\gamma_{2}\right)} \ll 1$, i.e., when $\gamma_{1}$ and $\gamma_{2}$ take large values. The lower bound in (53) is a harmonic mean of positive variables $\left(1+\gamma_{1}\right)$ and $\left(1+\gamma_{2}\right)$. Therefore, it can be further lower bounded as

$$
\tilde{\gamma} \geq \frac{1}{\frac{1}{\gamma_{1}+1}+\frac{1}{\gamma_{2}+1}} \geq \frac{1}{2} \min \left(\gamma_{1}+1, \gamma_{2}+1\right)
$$

where the second inequality turns to equality only if $\gamma_{1}=\gamma_{2}$. Due to the lower bound of (54), $P_{\mathrm{o}}$ can be upper bounded as

$$
\begin{aligned}
P_{\mathrm{o}} & =\operatorname{Pr}\left\{\frac{1}{2} \ln _{2}(\tilde{\gamma}) \leq r_{0}\right\} \\
& \leq \tilde{P}_{\mathrm{o}} \triangleq \operatorname{Pr}\left\{\frac{1}{2} \min \left(\gamma_{1}+1, \gamma_{2}+1\right) \leq 2^{2 r_{0}}\right\} .
\end{aligned}
$$

For a deterministic $b$ and independent random variables $X$ and $Y$, we know that $\operatorname{Pr}\{\min (X, Y) \leq b\}=1-\operatorname{Pr}\{\min (X, Y) \geq$ $b\}=1-\operatorname{Pr}\{X \geq b\} \operatorname{Pr}\{Y \geq b\}$. Thus, $\tilde{P}_{\mathrm{o}}$ is given by

$$
\begin{aligned}
\tilde{P}_{\mathrm{o}}= & 1-\operatorname{Pr}\left\{\gamma_{1} \geq t_{\mathrm{c}}\right\} \operatorname{Pr}\left\{\gamma_{2} \geq t_{\mathrm{c}}\right\} \\
= & \operatorname{Pr}\left\{\gamma_{1} \leq t_{\mathrm{c}}\right\}+\operatorname{Pr}\left\{\gamma_{2} \leq t_{\mathrm{c}}\right\} \\
& -\operatorname{Pr}\left\{\gamma_{1} \leq t_{\mathrm{c}}\right\} \operatorname{Pr}\left\{\gamma_{2} \leq t_{\mathrm{c}}\right\}
\end{aligned}
$$

where $t_{\mathrm{c}}=\nu 2^{2 r_{0}}-1$ and $\nu=2$. Based on (56), it is easy to see that

$$
P_{\mathrm{o}} \leq \operatorname{Pr}\left\{\gamma_{1} \leq t_{\mathrm{c}}\right\}+\operatorname{Pr}\left\{\gamma_{2} \leq t_{\mathrm{c}}\right\}
$$

where the bound is tight for large values of $\gamma_{1}$ and $\gamma_{2}$. Now, consider the following probability

$$
\operatorname{Pr}\left\{\sum_{n=1}^{L} \zeta_{n} x_{n} \leq t_{\mathrm{c}}\right\}
$$

where $x_{n}$ are i.i.d. exponentially distributed with mean 1 and $\zeta_{n} \geq 0$ for all $n=1, \ldots, L$ and $L$ is the number of $\left\{x_{n}\right\}$. For any $\beta>0$, the Chernoff bound [48] yields

$$
\begin{aligned}
\operatorname{Pr}\left\{\sum_{n=1}^{L} \zeta_{n} x_{n} \leq t_{\mathrm{c}}\right\} & \leq \mathrm{E}_{x}\left[\mathrm{e}^{-\beta\left(\sum_{n=1}^{L} \zeta_{n} x_{n}-t_{\mathrm{c}}\right)}\right] \\
& =\mathrm{e}^{\beta t_{\mathrm{c}}} \mathrm{E}_{x}\left[\mathrm{e}^{-\beta\left(\sum_{n=1}^{L} \zeta_{n} x_{n}\right)}\right] \\
& =\mathrm{e}^{\beta t_{\mathrm{c}}} \prod_{n=1}^{L} \mathrm{E}_{x_{n}}\left[\mathrm{e}^{-\beta \zeta_{n} x_{n}}\right] \\
& =\mathrm{e}^{\beta t_{\mathrm{c}}} \prod_{n=1}^{L} \frac{1}{1+\beta \zeta_{n}}
\end{aligned}
$$

where the second and third equalities are due to the facts that $x_{n}$ are statistically independent and $\mathrm{E}\left\{\mathrm{e}^{-\beta \zeta_{n} x_{n}}\right\}=$ $\int_{0}^{\infty} \mathrm{e}^{-\beta \zeta_{n} x_{n}} \mathrm{e}^{-x_{n}} d x_{n}$. Recalling that $\gamma_{1}=\frac{\alpha_{1}}{\sigma_{1}^{2}}$ and $\gamma_{2}=\frac{\alpha_{2}}{\sigma_{2}^{2}}$, where $\alpha_{1}$ and $\alpha_{2}$ are weighted sum of exponentially distributed random variables, after using (59) and (58), we obtain the upper bound of the OP given by (31). This completes the proof of Proposition 5.

\section{REFERENCES}

[1] J. Yang and S. Ulukus, "Optimal packet scheduling in an energy harvesting communication system," IEEE Trans. Commun., vol. 60, no. 1, pp. 220-230, Jan. 2012.

[2] K. Tutuncuoglu and A. Yener, "Optimum transmission policies for battery limited energy harvesting nodes," IEEE Trans. Wireless Commun., vol. 11, no. 3, pp. 1180-1189, Mar. 2012.

[3] B. Devillers and D. Gündüz, "A general framework for the optimization of energy harvesting communication systems with battery imperfections," J. Commun. Netw., vol. 14, no. 2, pp. 130-139, Apr. 2012.

[4] B. Devillers and Gündüz, "Two-hop communication with energy harvesting," in Proc. IEEE CAMSAP'11, San Juan, Puerto Rico, Dec. 2012, pp. 201-204.

[5] C. Huang, R. Zhang, and S. Cui, "Throughput maximization for the Gaussian relay channel with energy harvesting constraints," IEEE J. Sel. Areas Commun. Aug. 2013 [Online]. Available: http://arxiv.org/ pdf/1109.0724.pdf, preprint

[6] B. Gurakan, O. Ozel, J. Yang, and S. Ulukus, "Energy cooperation in energy harvesting wireless Communications," presented at the IEEE Int. Symp. Inf. Theory (ISIT) Boston, MA, USA, Jul. 2012.

[7] S. Luo, R. Zhang, and T. J. Lim, "Optimal save-then-transmit protocol for energy harvesting wireless transmitters," in Proc. IEEE Int. Symp. Inf. Theory (ISIT), Boston, MA, USA, Jul. 2012, pp. 955-959.

[8] R. Zhang and C. K. Ho, "MIMO broadcasting for simultaneous wireless information and power transfer," May 2011 [Online]. Available: http://arxiv.org/abs/1105.4999

[9] L. R. Varshney, "Transporting information and energy simultaneously," in Proc. IEEE Int. Symp. Inf. Theory (ISIT), Toronto, ON, Canada, Jul. 2008, pp. 1612-1616. 
[10] P. Grover and A. Sahai, "Shannon meets Tesla: Wireless information and power transfer," in Proc. IEEE Int. Symp. Inf. Theory (ISIT), Austin, TX, USA, Jun. 2010, pp. 2363-2367.

[11] A. M. Zungeru, L.-M. Ang, S. Prabaharan, and K. P. Seng, "Radio frequency energy harvesting and management for wireless sensor networks," [Online]. Available: http://arxiv.org/ftp/arxiv/papers/1208/1208.4439.pdf

[12] B. K. Chalise and L. Vandendorpe, "MIMO relay design for multipoint-to-multipoint communications with imperfect channel state information," IEEE Trans. Signal Process., vol. 57, no. 7, pp. 2785-2796, Jul. 2009.

[13] V. Tarokh, H. Jafharkani, and A. R. Calderbank, "Space-time block codes from orthogonal designs," IEEE Trans. Inf. Theory, vol. 45, no. 5, pp. 1456-1467, Jul. 1999.

[14] J. N. Laneman and G. W. Wornell, "Exploiting distributed spatial diversity in wireless networks," presented at the Allerton Conf. Commun., Cont. and Comput., Monticello, IL, USA, Oct. 2000.

[15] B. K. Chalise, Y. D. Zhang, and M. G. Amin, "Precoder design for OSTBC based AF MIMO relay system with channel uncertainty," IEEE Signal Process. Lett., vol. 19, no. 8, pp. 515-518, Aug. 2012.

[16] B. Khoshnevis, W. Yu, and R. Adve, "Grassmannian beamforming for MIMO amplify-and-forward relaying," IEEE J. Sel. Areas Commun., vol. 26, no. 8, pp. 1397-1407, Oct. 2008.

[17] S. M. Alamouti, "A simple transmit diversity technique for wireless communications," IEEE J. Sel. Areas Commun., vol. 16, no. 8, pp. $1451-1458$, Oct. 1998

[18] I. Hammerstrom and A. Wittneben, "Power allocation schemes for amplify-and-forward MIMO-OFDM relay links," IEEE Trans. Wireless Commun., vol. 6, no. 8, pp. 2798-2802, Aug. 2007.

[19] B. K. Chalise, Y. D. Zhang, and M. G. Amin, "Energy harvesting in an OSTBC based amplify-and-forward MIMO relay system," in Proc. IEEE ICASSP, Kyoto, Japan, Mar. 2012, pp. 3201-3204.

[20] D. Bültmann and T. Andre, "Analysis of 3GPP LTE-Advanced cell spectral efficiency," in Proc. IEEE 21st PIMRC, Istanbul, Turkey, Sep. 2010, pp. 1874-1879.

[21] J. I. Agbinya, Wireless Power Transfer, J. I. Agbinya, Ed. New York, NY, USA: River, 2012.

[22] U. Olgun, C.-C. Chen, and J. L. Volakis, "Investigation of rectenna array configurations for enhanced RF power harvesting," IEEE Antennas Propag. Lett., vol. 10, pp. 262-265, Apr. 5, 2011.

[23] X. Zhou, R. Zhang, and C. K. Ho, "Wireless information and power transfer: Architecture design and rate-energy tradeoff," in Proc. IEEE GLOBECOM'12, Anaheim, CA, Dec. 2012, pp. 1198-1203.

[24] A. Hjörugnes and D. Gesbert, "Precoding of orthogonal-space time block codes in arbitrarily correlated MIMO channels: Iterative and closed-form solutions," IEEE Trans. Wireless Commun., vol. 6, no. 3, pp. 1072-1082, Mar. 2007.

[25] A. Pascual-Iserte, D. P. Palomar, A. I. Perez-Neira, and M. A. Lagunas, "A robust Maximin approach for MIMO communications with imperfect channel state information based on convex optimization," IEEE Trans. Signal Process., vol. 54, no. 1, pp. 346-360, Jan. 2006.

[26] A. W. Marshall and I. Olkin, Inequalities: Theory of Majorization and Its Applications. New York, NY, USA: Academic, 1979.

[27] A. Abdel-Samad, T. N. Davidson, and A. B. Gershman, "Robust transmit eigen beamforming based on imperfect channel state information," IEEE Trans. Signal. Process., vol. 54, no. 5, pp. 1596-1609, May 2006.

[28] S. Boyd and L. Vandenberghe, Convex Optimization. Cambridge, U.K.: Cambridge Univ. Press, 2004.

[29] K. J. Arrow and A. C. Enthoven, "Quasi-concave programming," Econometrica, vol. 29, no. 4, pp. 779-800, Oct. 1961.

[30] Z.-Q. Luo, W.-K. Ma, A. M.-C. So, Y. Ye, and S. Zhang, "Semidefinite relaxation of quadratic optimization problems: From its practical deployments and scope of applicability to key theoretical results," IEEE Signal Process. Mag. (Special Issue on Convex Optimization for Signal Processing), vol. 27, no. 3, pp. 20-34, May 2010.

[31] Y. Huang and D. P. Palomar, "Rank-constrained separable semidefinite programming with applications to optimal beamforming," IEEE Trans. Signal Process., vol. 58, no. 2, pp. 664-678, Feb. 2010.

[32] M. Grant and S. Boyd, CVX: Matlab Software for Disciplined Convex Programming [Online]. Available: http://stanford.edu/ boyd/cvx

[33] S. Zhou and G. B. Giannakis, "Optimal transmitter eigen-beamforming and space-time block coding based on channel correlations," IEEE Trans. Inf. Theory, vol. 49, no. 7, pp. 1673-1690, Jul. 2002.
[34] M. Chiani, M. Z. Win, and A. Zanella, "On the capacity of spatially correlated MIMO Rayleigh fading channels," IEEE Trans. Inf. Theory, vol. 49, no. 10, pp. 2363-2371, Oct. 2003.

[35] R. A. Horn and C. R. Johnson, Topics in Matrix Analysis. Cambridge, U.K.: Cambridge Univ. Press, 1991.

[36] T. Q. Duong, G. C. Alexandropoulos, H.-J. Zepernick, and T. A. Tsiftsis, "Orthogonal space-time block codes with CSI-assisted amplify-and-forward relaying in correlated Nakagami- $m$ fading channels," IEEE Trans. Veh. Technol., vol. 60, no. 3, pp. 882-889, Mar. 2011

[37] K. Yang, J. Yang, and L. Cai, "Dual-hop MIMO relaying with OSTBC over doubly-correlated Nakagami-m fading channels," EURASIP $J$ Wireless Commun. Netw. Sep. 2012 [Online]. Available: http://jwcn. eurasipjournals.com/content/pdf/1687-1499-2012-294.pdf

[38] J.-B. Kim and D. Kim, "Comparison of tightly power-constrained performances for opportunistic amplify-and-forward relaying with partial or full channel information," IEEE Commun. Lett., vol. 13, no. 2, pp. 100-102, Feb. 2009.

[39] X. Zhang, D. P. Palomar, and B. Ottersten, "Statistically robust design of linear MIMO transceivers," IEEE Trans. Signal Process., vol. 56, no. 8, pp. $3678-3689$, Aug. 2008.

[40] L. Grippo and M. Sciandrone, "On the convergence of the block nonlinear Gauss-Seidel method under convex constraints," Operat. Res. Lett., vol. 26, pp. 127-136, 2000.

[41] S. Kandukuri and S. Boyd, "Optimal power control in interference-limited fading wireless channels with outage-probability specifications," IEEE Trans. Wireless Commun., vol. 1, no. 1, pp. 46-55, Jan. 2002.

[42] M. Bengtsson and B. Ottersten, "Signal waveform estimation from array data in angular spread environment," in Proc. 13th Asilomar Conf. Signal Syst. Comput., Pacific Grove, CA, USA, Nov. 1996, vol. 1, pp. 355-359.

[43] B. K. Chalise and L. Vandendorpe, "A multi-user MIMO transmit beamformer based on the statistics of the signal-to-leakage ratio," EURASIP J. Wireless Commun. Netw., vol. 2009, 679430.

[44] M. Bengtsson and B. Ottersten, "Optimum and suboptimum transmit beamforming," in Handbook of Antennas in Wireless Communications. Boca Raton, FL, USA: CRC, 2002.

[45] M. R. Mckay, A. J. Grant, and I. B. Collings, "Performance analysis of MIMO-MRC in double-correlated Rayleigh environments," IEEE Trans. Commun., vol. 55, no. 5, pp. 497-507, Mar. 2007.

[46] D. R. Cox, Renewal Theory. London, 1967, Methuens Monographs on Appl. Probabil. Statist..

[47] I. S. Gradshteyn and I. M. Ryzhik, Table of Integrals, Series, and Products. New York, NY, USA: Academic , 2000.

[48] M. K. Simon and M.-S. Alouini, Digital Communications Over Fading Channels 2004, Wiley Series in Telecommun. and Signal Process.

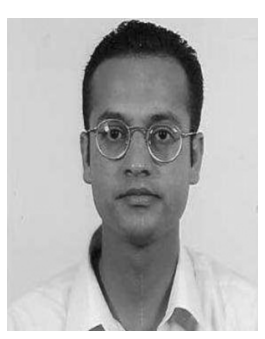

Batu Krishna Chalise (SM'11) received the B.E. degree in electronics engineering from Tribhuvan University, Kathmandu, Nepal, in 1998 and the M.S. and $\mathrm{Ph} . \mathrm{D}$. degrees in electrical engineering from the University of Duisburg-Essen, Duisburg, Germany, in 2001 and 2006, respectively.

In 1999, he was a lecturer with the Institute of Engineering, Kathmandu. For a short period in 2001, he was a Researcher with the Fraunhofer-Institute of Microelectronic Circuits and Systems (IMS), Duisburg. From 2002 to 2006, he was a Research Assistant with the Department of Communication Systems, University of Duisburg-Essen, where his Ph.D. research was supported by a grant from the Ministry of Education and Science of North Rhein-Westphalia (NRW), Germany. He was a Postdoctoral Researcher with the Communication and Remote Sensing Laboratory, Universitè catholique de Louvain, Louvain La Neuve, Belgium from December 2006 till June 2010. Currently, he is the Postdoctoral Research Fellow with the Center for Advanced Communications, Villanova University, Villanova, PA. His research interests include cooperative and opportunistic wireless communications, robust algorithms for multiantenna systems, convex optimization, unmanned aerial vehicle networks, and passive radar systems.

Dr. Chalise is an Associate Editor of the EURASIP Journal of Wireless Communications and Networking. 


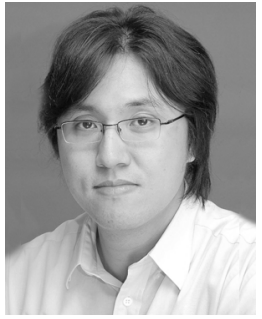

Wing-Kin Ma (M'01-SM'11) received the B.Eng. (first-class honors) degree in electrical and electronic engineering from the University of Portsmouth, Portsmouth, U.K., in 1995, and the M.Phil. and $\mathrm{Ph} . \mathrm{D}$. degrees, both in electronic engineering, from The Chinese University of Hong Kong (CUHK), Hong Kong, in 1997 and 2001, respectively.

$\mathrm{He}$ is currently an Assistant Professor with the Department of Electronic Engineering, CUHK. From 2005 to 2007, he was also an Assistant Professor with the Institute of Communications Engineering, National Tsing Hua University, Taiwan, R.O.C., where he is still holding an adjunct position. Prior to becoming a faculty member, he held various research positions with McMaster University, Canada; CUHK; and the University of Melbourne, Australia. His research interests are in signal processing and communications, with a recent emphasis on MIMO communication, convex optimization, and blind signal processing.

Dr. Ma is currently serving or has served as Associate Editor and Guest Editor of several journals, which include IEEE TRANSACTIONS ON SIGNAL Processing, IEEE Signal Processing LetTers, IEEE Journal of Selected areas in Communications and IEEE Signal Processing MAgazinE. He is a Member of the Signal Processing Theory and Methods (SPTM) Technical Committee. His student has won an ICASSP 2011 Best Student Paper Award, and he is corecipient of a WHISPERS 2011 Best Paper Award.

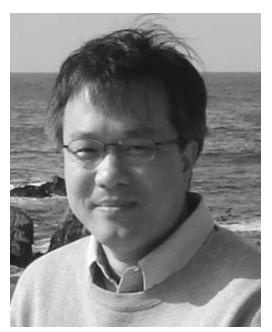

Yimin D. Zhang (SM'01) received the Ph.D. degree from the University of Tsukuba, Tsukuba, Japan, in 1988.

He joined the faculty of the Department of Radio Engineering, Southeast University, Nanjing, China, in 1988. He served as a Technical Manager at the Communication Laboratory Japan, Kawasaki, Japan, from 1995 to 1997, and was a Visiting Researcher at ATR Adaptive Communications Research Laboratories, Kyoto, Japan, from 1997 to 1998. Since 1998, he has been with the Villanova University, Villanova, PA, where he is currently a Research Professor with the Center for Advanced Communications and the Director of the Wireless Communications and Positioning Laboratory. He has more than 200 publications in the area of statistical signal and array processing for communications and radar applications, including wireless networks, cooperative communications, MIMO systems, source localization and target tracking, over-the-horizon radar, passive sensing, jammer suppression, time-frequency analysis, radio frequency identification (RFID), and image processing.

Dr. Zhang is an Associate Editor for the Journal of the Franklin Institute and an Editor for the Signal Processing journal. He is an Associate Editor for the IEEE TRANSACTIONS ON SIGNAL PROCESSING and was an Associate Editor for the IEEE SignAL PROCESSING LeTters from 2006 to 2010.

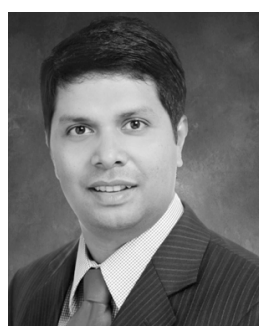

Himal A. Suraweera (S'04-M'07) received the B.Sc. degree (first class honors) in electrical and electronics engineering Peradeniya University, Peradeniya, Sri Lanka, in 2001 and the Ph.D. degree from Monash University, Melbourne, Australia, in 2007.

From 2001 to 2002, he was with the Department of Electrical and Electronics Engineering, Peradeniya University, as an Instructor. From October 2006 to January 2007, he was at Monash University as a Research Associate. From February 2007 to June 2009, he was at the Center for Telecommunications and Microelectronics, Victoria University, Melbourne, as a Research Fellow. From July 2009 to January 2011, he was with the Department of Electrical and Computer Engineering, National University of Singapore, Singapore as a Research Fellow. From January 2011 to May 2013, he was a Postdoctoral Research Associate at the Singapore University of Technology and Design. He is currently a Senior Lecturer at the Department of Electrical and Electronic Engineering, University of Peradeniya, Peradeniya, Sri Lanka. His main research interests include relay networks, wireless security, Green communications, cognitive radio, and MIMO.

Dr. Suraweera is an Associate Editor of the IEEE COMMUNICATIONS LetTERs. He received an IEEE COMmUniCATIONS LetTERS Exemplary Reviewer Certificate in 2009, an IEEE Communications Society Asia-Pacific Outstanding Young Researcher Award in 2011 and an IEEE WIRELESS COMMUNICATIONS LeTTERs Exemplary Reviewer Certificate in 2012. He was a recipient of an International Postgraduate Research Scholarship from the Australian Commonwealth during 2003-2006. He was also awarded the 2007 Mollie Holman Doctoral and 2007 Kenneth Hunt Medals for his doctoral thesis upon graduating from Monash University.

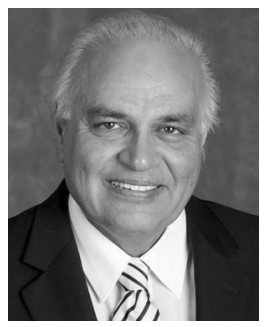

Moeness G. Amin (F'01) received the Ph.D. degree in 1984 from University of Colorado, in electrical engineering.

He has been on the Faculty of the Department of Electrical and Computer Engineering at Villanova University, Villanova, PA, since 1985. In 2002, he became the Director of the Center for Advanced Communications, College of Engineering. He has over 450 journal and conference publications in the areas of wireless communications, time-frequency analysis, smart antennas, waveform design and diversity, interference cancellation in broadband communication platforms, anti-jam GPS, target localization and tracking, direction finding, channel diversity and equalization, ultrasound imaging, and radar signal processing.

Dr. Amin is the Recipient of the 2009 Individual Technical Achievement Award from the European Association of Signal Processing, and the Recipient of the 2010 NATO Scientific Achievement Award. He is a Fellow of the International Society of Optical Engineering, 2007; and a Fellow of the Institute of Engineering and Technology (IET), 2010. He is a Recipient of the IEEE Third Millennium Medal, 2000; Recipient of the Chief of Naval Research Challenge Award, 2010; Distinguished Lecturer of the IEEE Signal Processing Society, 2003-2004; Active Member of the Franklin Institute Committee on Science and the Arts; Recipient Villanova University Outstanding Faculty Research Award, 1997; and the Recipient of the IEEE Philadelphia Section Award, 1997. He is a member of SPIE, EURASIP, ION, Eta Kappa Nu, Sigma Xi, and Phi Kappa Phi. He currently serves on the Overview Board of the IEEE TRANSACTIONS ON Signal Processing. He also serves on the Editorial Board of the EURASIP Signal Processing Journal. He was a Plenary Speaker at ICASSP 2010. He was the Special Session Co-Chair of the 2008 IEEE International Conference on Acoustics, Speech, and Signal Processing. He was the Technical Program Chair of the 2nd IEEE International Symposium on Signal Processing and Information Technology, 2002. He was the General and Organization Chair of the IEEE Workshop on Statistical Signal and Array Processing, 2000. He was the General and Organization Chair of the IEEE International Symposium on Time-Frequency and Time-Scale Analysis, 1994. He was an Associate Editor of the IEEE TRANSACTIONS ON Signal PROCESSING during 1996-1998. He was a member of the IEEE Signal Processing Society Technical Committee on Signal Processing for Communications during 1998-2002. He was a Member of the IEEE Signal Processing Society Technical Committee on Statistical Signal and Array Processing during 1995-1997. He has given several keynote and plenary talks, and served as a Session Chair in several technical meetings. He was the Guest Editor of the Journal of Franklin Institute September 2008 Special Issue on Advances in Indoor Radar Imaging. He was a Guest Editor of the IEEE Transactions on Geoscience and Remote Sensing May 2009 Special Issue on Remote Sensing of Building Interior, and a Guest Editor of the ET Signal Processing December 2009 Special Issue on Time-Frequency Approach to Radar Detection, Imaging, and Classification. 